\title{
La fable des poissons de Cyrus (Hérodote, I, 141) [Son origine et sa fonction dans l'économie des Histoires d'Hérodote] \\ Son origine et sa fonction dans l'économie des Histoires d'Hérodote
} Paola Ceccarelli

\section{Résumé}

La fable des poissons de Cyrus (Hérodote, 1, 141): son origine et sa fonction dans l'économie des Histoires (pp. 29-57) L'auteur se propose d'évaluer le rapport entre l'organisation narrative des Histoires et le "réel" tel qu'il a pu se présenter à Hérodote, en prenant comme point de départ un segment précis du texte, l'apologue adressé par Cyrus aux loniens (Hérodote, $1,141)$. Une réponse à la question de l'existence de référents historiques est apportée par la présence d'éléments parallèles, aussi bien dans la tradition grecque que dans la tradition perse. D'autre part, une analyse interne au texte, soulignant le rôle de l'apologue de Cyrus dans la structure générale des Histoires, permet de donner un sens au choix d'Hérodote d'insérer justement cet apologue, sous cette forme, dans sa narration.

\section{Citer ce document / Cite this document :}

Ceccarelli Paola. La fable des poissons de Cyrus (Hérodote, I, 141) [Son origine et sa fonction dans l'économie des Histoires d'Hérodote]. In: Mètis. Anthropologie des mondes grecs anciens, vol. 8, n¹-2, 1993. pp. 29-57;

doi : $10.3406 /$ metis. 1993.990

http://www.persee.fr/doc/metis_1105-2201_1993_num_8_1_990

Document généré le 10/04/2016 


\section{LA FABLE DES POISSONS DE CYRUS (HÉRODOTE, I, 141) Son origine et sa fonction dans l'économie des Histoires d'Hérodote}

Immédiatement après la conquête perse de la Lydie, en 546 av. J.-C. environ, les Ioniens et les Éoliens envoyèrent des ambassadeurs à Sardes, pour négocier avec Cyrus un accord aux mêmes conditions qu'avec Crésus quelques années auparavant ${ }^{1}$. A propos de cette ambassade Hérodote parle de "Ioniens et Éoliens" en général; tous les Grecs d'Asie Mineure sont donc concernés, indépendamment de leur situation géographique. Cyrus refuse de négocier et répond aux Ioniens par une fable célèbre, l'apologue du

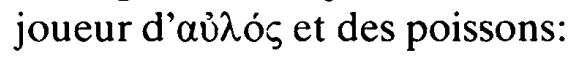

Cyrus, après avoir écouté leurs propositions, leur conta cette fable $(\ddot{\varepsilon} \lambda \varepsilon \xi \dot{\varepsilon}$ $\sigma \phi \iota \lambda$ ó $\left.{ }^{\circ} \circ\right)$ : "Un joueur de flûte", dit-il, "voyant des poissons dans la mer, jouait de son instrument; il pensait qu'ils sortiraient de l'eau et viendraient sur la terre. Déçu dans son espérance, il saisit un filet ( $\lambda \alpha 6 \varepsilon i ̃ v ~ \grave{\alpha} \mu \phi i-$ $6 \lambda \eta \sigma \tau \rho \circ v)$, enveloppa une grande masse de poissons, les tira hors de la mer; et les voyant sauter ( $\pi \alpha \lambda \lambda \mathrm{o} \mu \varepsilon \dot{\varepsilon} v 0 u \varsigma$ ), il leur dit: Cessez-moi ces danses, puisque, quand je jouais de la flûte, vous ne vouliez pas même sortir de l'eau".

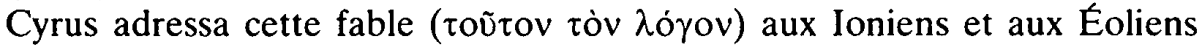
parce qu'auparavant, quand lui-même les avait fait prier par ses députés de

* Je tiens à exprimer toute ma reconnaissance à Claude Calame, qui a suivi les différentes étapes de ce travail et m'a fait profiter de ses remarques; Justin Favrod, Martin Steinrück et Pierre Voelke m'ont aussi apporté de précieuses suggestions: qu'ils en soient tous remerciés.

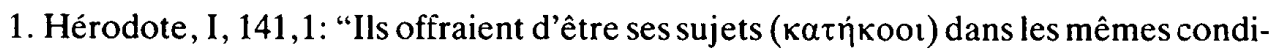
tions qu'ils l'avaient été de Crésus"; cela aurait signifié la soumission pour tous les Grecs de la côte, I 6,2; quant aux insulaires, Crésus avait conclu avec eux un accord, ร̌દivin, I, 27,5 . 
se détacher de Crésus, les Ioniens n'avaient pas écouté, et qu'ils étaient prêts à l'écouter alors, quand l'affaire était terminée 2 .

Résultat immédiat de cet apologue: les Ioniens rentrent chez eux et élèvent des enceintes fortifiées autour de leurs villes; ils se réunissent ensuite au Panionion pour organiser la défense, tous sauf les Milésiens, car avec eux Cyrus a conclu un traité aux mêmes conditions que Crésus. Là aussi Hérodote souligne nettement le fait que tous les Ioniens, sauf les Milésiens, se réunirent au Panionion et qu'ils décidèrent ensemble d'envoyer des messagers demander de l'aide à Sparte ${ }^{3}$.

Par la suite, Hérodote va distinguer entre Grecs des îles et Grecs de la côte; lors de la digression sur les Ioniens et les Éliens (I, 1+2-151), il précise pour chaque cité son emplacement géographique, sur la côte ou sur une île, et à deux reprises, au début et à la conclusion de sa digression, il souligne que les insulaires pensaient n'avoir rien à craindre, puisque les Perses n'avaient pas de flotte ${ }^{\dagger}$. Néanmoins, la situation pour les insulaires a aussi changé depuis l'avènement de Cyrus; en effet, l'accord qu'ils avaient avec Crésus n'a pas été renouvelé, leur relative sécurité est due au seul fait qu'ils pensent être hors d'atteinte. On comprend de ce fait mieux pourquoi, après avoir relaté la soumission des Ioniens par Harpagos, Hérodote ajoute que les Ioniens des îles, effrayés par les événements, se rendirent à Cyrus ${ }^{5}$. En fait, les insulaires sont, tout autant que les autres

2. Hérodote, I, 141, 1-2; Cyrus avait effectivement envoyé auparavant des hérauts auprès des Ioniens pour les solliciter de se détacher de Crésus, I 76.3. Les traductions de passages d'Hérodote sont tirées de Ph.-E. Legrand, Hérodote. Histoires, Paris 1932-54; pour le texte d'Hérodote $\mathrm{j}$ 'ai suivi l'édition de C. Hude. Herodoti Historiae, Oxford ${ }^{3}$ 1927. Sur la fable dans l'antiquité, voir T. Karadagli, Fabel und Ainos. Studien zur griechischen Fabel, Königstein/Ts. 1981, pour celle-ci en part. p. 23 et p. 65, ct les articles réunis dans le volume La fable, Entretiens Hardt XXX. Genève 1984. Pour une bonne discussion des "fables perses" dans les Histoires d'Hérodote voir K. Reinhardt, "Herodots Persergeschichten", in K. Reinhardt, Von Werken und Formen, Godesberg 1948, pp. 163-224 (repris ensuite in C. von Becker éd., Vermächtnis der Antike, Göttingen 1960, pp. 133-174).

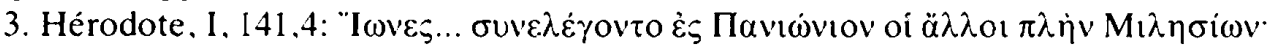

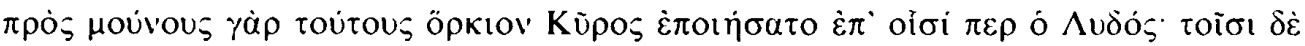

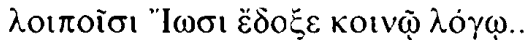

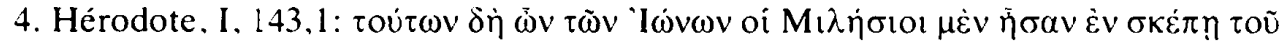

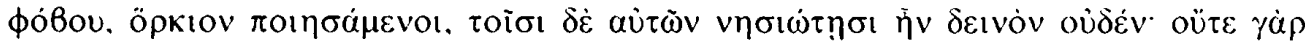

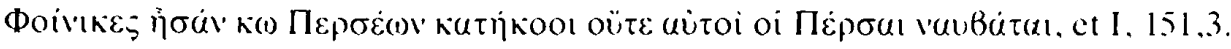

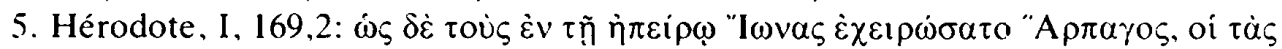

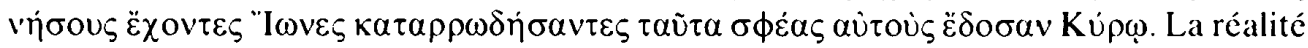
de cette $\delta i \delta \omega \sigma ı \varsigma$ a été mise en question, notamment par L. Boffo, "La conquista persiana 
habitants de la côte, concernés par la réponse de Cyrus, et l'apologue se réalise très vite dans la narration avec la soumission totale des Grecs d'Asie Mineure, insulaires et habitants de la côte.

Il est possible de se poser, à propos de cet apologue, deux sortes de questions, qui renvoient à deux démarches différentes. En effet, s'agissant de l'analyse du texte d'un historien, on peut choisir de poser le problème du degré d'historicité de l'anecdote ${ }^{6}$, de l'existence ou non de référents historiques; d'autre part, puisque ce texte est organisé dans une configuration narrative, on peut se demander quelle est la fonction de cette anecdote dans le récit d'Hérodote.

Dans le premier cas, la démarche consiste à rechercher des éléments parallèles au texte d'Hérodote dans la tradition grecque et dans la tradition perse. En effet, si la comparaison entre des peuples vaincus et des poissons est présente dans la tradition perse antérieure à Hérodote, il est possible de penser qu'Hérodote nous rapporte ici fidèlement les propos de Cyrus; en revanche, si l'on ne trouve des parallèles que dans la tradition grecque, on pourra penser à une "manipulation"7 de la part d'Hérodote. manipulation qu'il faudra ensuite essayer d'expliquer. S.W. Hirsch a récemment proposé une analyse de ce type, portant sur l'historicité de l'épisode (Cyrus a-t-il réellement prononcé cette fable?) et sur l'origine, grecque ou orientale, de la comparaison entre peuples maritimes vaincus et poissons ${ }^{\gamma}$. Une telle démarche est sans doute intéressante: au-delà d'in-

delle città greche d'Asia minore", Monumenti antichi pubblicati dall'Accademia dei Lincei, 26, 1983, p. 45 et $n$. 227. Il n'y a aucune raison de refuser la donnée hérodotéenne; de toute façon, cela n'a pas d'importance pour notre étude.

6. Par "degré d'historicité" jentends ici (et dans le reste du travail) le niveau de prétention référentielle directe à la "vérité" telle qu on peut l'établir selon les règlès de lévidence commune à tout le corps des sciences. Voir à ce propos, ainsi que sur les ambiguités rattachées au terme même d" "histoire" (et done d"“historicité"). P. Ricoeur. "Lat fonction narrative", in D. Tiffeneau éd., La Narrativité, Paris 1980, pp. 49-68. en part. p. 58.

7. Gr. Nagy, Pindar's Homer, Baltimore-London, 1991, pp. 324-325 et n. 59, pense qu'il s'agit d'une fable grecque attribuée par Hérodote aux Perses et parle à ce propos de "manipulation": il cite comme autre exemple d'un discours grec attribué aux Perses ke Jébat sur les constitutions. Hérodote, III, 80-88. Sur la difficulté de distinguer entre réits perses et récits attribués aux Perses par les Grecs, voir les remarques de O. Murray. "Herodotus and Oral History", in H. Sancisi-Weerdenbug et A. Kuhrt éd. . Achaemenid History II, Leiden 1987. pp. 93-115. en part. pp. 108-115, ainsi que K. Reinhardt, art. cit.

8. S.W. Hirsch, "Cyrus' Parable of the Fish: Sea Power in the Early Relations of ireece and Persia", Classical Journal, 81, 1985-86, pp. 222-229. Hirsch conclut, sur la ase des parallèles orientaux, à l'historicité de l'épisode; il propose ensuite une lecture 
dications possibles quant à l'existence d'un référent pour le texte en question, et donc quant à son historicité éventuelle, elle permet de reconstituer le contexte dans lequel se place le texte d'Hérodote, ce qu'on peut définir comme l'horizon d'attente ${ }^{9}$ du public auquel s'adressent les Histoires. Délimiter l'horizon d'attente d'Hérodote et de ses contemporains a son importance pour l'analyse du texte des Histoires; en effet, d'une part Hérodote a certainement dû en tenir compte pour assurer la compréhension de son œuvre; d'autre part, on peut, en se référant à cet horizon d'attente, mesurer les écarts du texte des Histoires par rapport à ce cadre et donc définir ce qui est plus spécifiquenent propre au discours d'Hérodote. Cependant, une telle démarche n'est pas suffisante: montrer que l'image utilisée dans la réponse de Cyrus aux Ioniens a des précédents grecs ou perses (nous verrons qu'ils sont grecs et perses) ne permet de toute façon pas d'attribuer à cette réponse un statut de "vérité" au sens positiviste du terme; cela ne suffit pas non plus à justifier le choix d'Hérodote de l'insérer, sous la forme particulière qu'elle revêt, dans les Histoires.

Seule une analyse interne au texte d'Hérodote permet de comprendre la fonction de cet apologue, en mettant en relief les rapports qu'il entretient avec l'ensemble du texte. Cette seconde démarche peut trouver sa justification en elle-même; mais je voudrais montrer par un cas précis que les deux démarches peuvent s'intégrer l'une à l'autre, et que ce n'est qu'en les intégrant qu'il est possible de rendre justice à la multiplicité des significations d'un texte comme celui d'Hérodote. Dans l'écriture de l'histoire se pose en effet de façon particulièrement aiguë le problème du rapport entre d'une part l'organisation du récit, la mise en intrigue donnant aux événements leur signification, et d'autre part le contenu du récit, les événements ou les informations, qui constituent le matériau sur lequel l'historien s'est

\footnotetext{
de cette parabole en termes d'opposition structurale entre terre et mer, et définit par ce biais le rôle de la fable du flûtiste et des poissons dans les Histoires. D'autres passages sont plus pertinents à ce propos (notamment I 27, cité aussi par Hirsch; je renvoie à mon article "De la Sardaigne à Naxos: le rôle des îles dans les Histoires d'Hérodote", F. Létoublou (éd.), Impressions d'îles, Toulouse 1996, pp. 41-54. Une opposition binaire Grecs-marins / Perses-continentaux est excessivement simplificatrice: une grande partie des problèmes de l'Ionie est due au fait qu'il y avait des Grecs établis justement sur le continent; j’ai utilisé moi-même par la suite la métaphore des "poissons" pour indiquer les Grecs insulaires; il ne faut toutefois pas oublier que ceux-ci se trouvent sur une terre, même si elle est insulaire.

9. Pour l'utilité de la notion d'horizon d'attente et pour ses retombées dans l'analyse même du texte, voir P. Payen, "Discours historique et structures narratives chez Hérodote", Annales ESC 45, 3, 1990, pp. 529-531.
} 
basé pour construire son récit ${ }^{10}$. Il s'agit d'un rapport qui est souvent conçu en termes conflictuels, ce qui a pour conséquence la valorisation excessive de l'un des deux aspects au détriment de l'autre. L'analyse qui suit se propose comme but d'évaluer, pour un segment précis du texte d'Hérodote, le rapport entre l'organisation narrative des Histoires et le "réel" tel qu'il a pu se présenter à Hérodote, et tel que celui-ci a pu vouloir le transmettre à son public.

Nous allons donc procéder à une analyse sur deux niveaux différents, répondant à trois questions précises:

1) La fable du flûtiste et des poissons est-elle attestée dans la tradition grecque antérieure à Hérodote, et à quoi renvoie, dans la tradition grecque, l'image de poissons pris au filet?

2) Pourquoi Hérodote a-t-il attribué cette fable justement à un Perse? Y a-t-il des parallèles orientaux pour la comparaison entre la soumission de populations côtières et la pêche de poissons?

3) Enfin, et surtout, quel est le rôle de cette fable dans le texte d'Hérodote? Quelle est sa signification dans les Histoires?

Nous insistons sur le fait que la recherche de parallèles externes au texte d'Hérodote n'a pas pour but d'établir, au moyen de la concordance entre plusieurs sources, une "vérité absolue", mais simplement de circonscrire un cadre de "vérité possible" auquel Hérodote aurait pu avoir accès et à l'intérieur duquel il aurait pu choisir ce qu'il voulait conserver dans sa narration. Ces parallèles, puisqu'ils nous servent seulement à établir un cadre de référence et qu'ils ne constituent pas en eux-mêmes l'objet de notre analyse, seront relativement peu ou pas replacés en contexte.

\section{Parallèles dans la tradition grecque}

\subsection{La fable}

L'histoire du flûtiste et des poissons dansants est attestée sous une forme très proche dans le corpus des fables d'Ésope ainsi que dans les recueils de fables de Babrius et d'Aphthonios ${ }^{11}$; ces derniers ont été composés à

10. Cf. les questions soulevées par N. Loraux, dans le compte rendu de F. Hartog, "Le miroir d'Hérodote", Annales ESC, 1982, 3, pp. 493-497, et P. Ricœur, "L'histoire comme récit", in D. Tiffeneau (éd.), La Narrativité, Paris 1980, pp. 5-24. W.K. Pritchett, The Liar School of Herodotos, Amsterdam, 1993, a récemment pris position-d'une façon discutable- en faveur de la "véracité" d'Hérodote; voir à ce sujet le long compte rendu de N. Luraghi, Quaderni di Storia, 40, 1994, pp. 181-190.

11. B.E. Perry, Aesopica I, Urbana 1952, n. 11=24 Chambry; B.E. Perry, Babrius and Phaedrus, London 1965, n. 9=9 Luzzatto-La Penna, Babrius Mythiambi Aesopei, 
l'époque romaine, le recueil de Babrius entre la fin du $\mathrm{I}^{\mathrm{er}}$ et le début du $\mathrm{II}^{\mathrm{e}}$ siècle de notre ère, celui du rhéteur Aphthonios entre le $I V^{e}$ et le $V^{e}$ siècle de notre ère. Quant à Ésope, si la tradition nous dit qu'il vécut à Samos au $\mathrm{VI}^{\mathrm{e}}$ siècle av. J.-C., la première collection de ses fables, celle de Démétrios de Phalère, remonte au début de l'époque alexandrine, et la première recension qui nous soit parvenue, l'Augustana, dans laquelle se trouve notre fable, ne remonte qu'au $\mathrm{I}^{\text {er }}$ siècle de notre ère ${ }^{12}$. La fable du flûtiste et des poissons semble avoir été assez populaire au $\mathrm{I}^{\mathrm{er}}$ siècle, puisqu'on en trouve un écho dans les Évangiles ${ }^{13}$; elle réapparaît encore au III $^{\mathrm{e}}$ siècle de notre ère dans la Vie d'Apollonios de Tyane de Philostrate ${ }^{14}$. Il semble certain que Philostrate pour ce chapitre s'inspire d'Hérodote ${ }^{15}$.

L'attestation la plus ancienne de cette fable, en dehors du texte d'Hérodote, se trouve donc dans le corpus ésopique. Voici le texte de la fable ${ }^{16}$ :

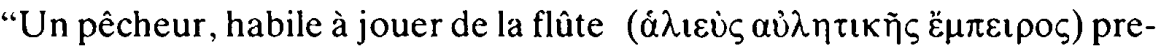

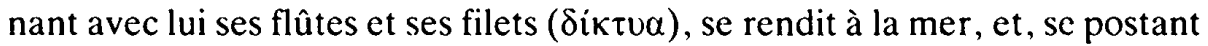
sur un rocher en saillie, il se mit d'abord à jouer, pensant que les poissons, attirés par la douceur de ses accords, allaient d'eux-mêmes sauter hors de l'eau pour venir à lui. Mais comme, en dépit de ses longs efforts, il n'en était pas plus avancé, il mit de côté ses flûtes, prit son épervier $(\dot{\alpha} \mu \phi i B \lambda \eta \sigma \tau \rho o v)$, et, le

Leipzig, 1986; Aphthonios, in A. Hausrath, Corpus Fabularum Aesopicarum I.2, Leipzig 1911, p. 148 n. 33.

12. Cf. B.E. Perry, Introduction to Babrius and Phaedrus, Cambridge Mass, 1965, XVI-XVII. La classe I de l'Augustana est indépendante de la tradition de Babrius, mais un certain nombre de ses fables, dont la nôtre, dérivent d'une source commune à la classe I et à Babrius; cette source pourrait être l'Aesopia de Démétrios. Sur la tradition des fables ésopiques, cf. en dernier S. Jedrkiewicz, Sapere e paradosso nell'Antichità: Esopo e la favola, Roma 1989, pp. 15-37; sur leur origine orientale, voir M.L. West, "The Ascription of Fables to Aesop in Archaic and Classical Greece", in La Fable cit., pp. 105128.

13. Matthieu, 11, 16-17: "A qui vais-je comparer cette génération? Elle est comparable à des enfants assis sur les places, qui en interpellent d'autres: Nous-vous avons joué de la flûte ct vous n'avez pas dansé! Nous avons entonné un chant funèbre, et vous ne vous êtes pas frappé la poitrine"; même comparaison chez Luc 7, 32 .

14. I, 23; davantage sur ce passage infra. Un exemple de pêche par la musique dans Élien, De la nature des animaux, I 39.

15. Et non de Ctésias comme l'affirme Hirsch, art.cit., p. 225, en interprétant mal l'article de F. Grosso, "Gli Ateniesi deportati ad Eretria", Rivista di filologia e di istruzione classica, 36, 1958, pp. 350-75; Ctésias, comme l'a montré Grosso, est la source de Philostrate pour le ch. 24 de la Vie d'Apollonios; dans le ch. 24 on parle aussi, en effet, des Érétriens, mais en termes contradictoires par rapport à la narration même de Philostrate en I 23 et à celle d'Hérodote.

16. Dans la traduction de E. Chambry, Ésope. Fables, Paris, 1927, n. 24. 


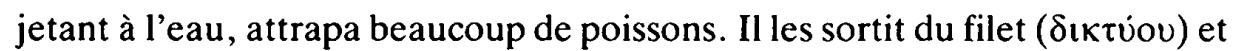
les jeta sur le rivage; et, comme il les voyait frétiller ( $\sigma \pi \alpha i \rho o v \tau \alpha \varsigma)$, il s'écria: "Maudites bêtes, quand je jouais de la flûte, vous ne dansiez pas ( $\omega \rho \chi \varepsilon i ̃ \sigma \theta \varepsilon)$; à présent que j'ai fini, vous vous mettez en branle". Cette fable s'applique à ceux qui agissent à contre-temps".

Il est à notre avis impossible de tirer d'une comparaison entre fable ésopique et texte d'Hérodote des renseignements sur le rapport entre les deux versions; tout au plus peut-on remarquer qu'Ésope et Babrius com-

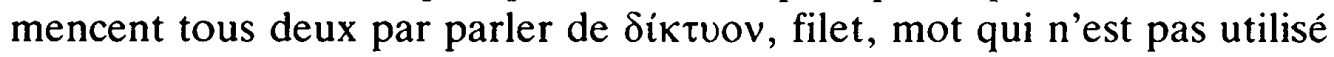
chez Hérodote; mais dès qu'on entre dans le déroulement de l'anecdote, Babrius utilise $\sigma \alpha \gamma \dot{\eta} v \eta$ (je montrerai plus loin en quoi ce terme est en rapport avec l'anecdote des poissons) et Ésope $\alpha \mu \phi i B \lambda \eta \sigma \tau \rho o v$, le terme employé dans ce contexte par Hérodote, quitte à revenir ensuite, deux lignes

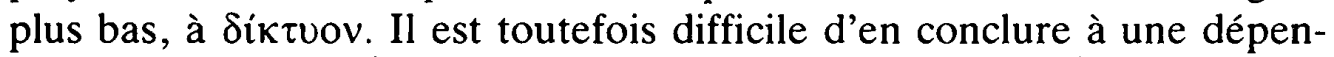
dance de la fable d'Ésope par rapport au texte d'Hérodote ${ }^{17}$.

En revanche, il y a certainement, comme l'a souligné $\mathrm{Nagy}^{18}$, une ressemblance du point de vue de la forme entre les fables d'Ésope et le discours d'Hérodote; en effet ce dernier choisit souvent de s'exprimer en utilisant la forme caractéristique de l'alvos. Il est par conséquent possible que cette fable ait figuré dans la tradition grecque au moment de la composition des Histoires, et qu'Hérodote l'ait intégrée à son texte; n'oublions pas qu'Hérodote lui-même, dans un autre passage de son œuvre (II 134,3),

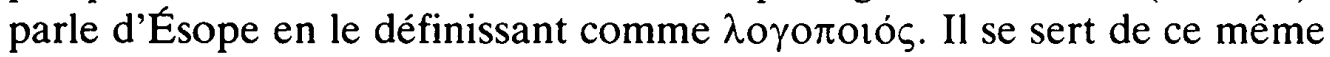
terme pour désigner son propre prédécesseur Hécatée (II 143,1), ce qui implique qu'il voit une parenté entre son discours et celui d'Ésope; en outre, une telle définition implique la connaissance de sa part d'un certain nombre de $\lambda$ ó $\gamma o r$ ésopiques. Enfin, ce que nous appellerons l'aīvos des poissons est désigné par le narrateur à deux reprises, en introduction et en conclusion, par le terme $\lambda$ ó $\gamma \circ$, terme très général qui caractérise chez Hérodote également le discours ésopique.

On ne peut donc affirmer aussi catégoriquement que le fait Hirsch $^{19}$

17. Pour compliquer le problème, la morale de la fable de Babrius n'a aucun rapport avec celle qui ressort du texte d'Hérodote ni avec la morale ésopique; elle est en revanche proche de celle de la version d'Aphthonios.

18. Nagy, op.cit., pp. 314-338, en part. pp. 324-325 et p. 335, et G. Nagy, "Mythe et prose en Grèce archaïque: l'ainos", in C. Calame (éd.), Métamorphoses du mythe en Grèce antique, Genève, 1988, pp. 229-242; plus généralement sur le rôle de l'ainos dans la Grèce archaïque, Nagy, op.cit., pp. 214-338. Pour la signification à attribuer au fait yue la réponse de Cyrus présente la structure formelle de l'ainos, voir infra.

19. Art.cit., pp. 225-226. 
qu'il n'existe aucune version grecque de cet apologue qui soit antérieure à Hérodote et qui pourrait servir à prouver une origine grecque de l'aĩvos des poissons dansants.

\subsection{La comparaison entre hommes vaincus et poissons pris au filet}

Au-delà d'éventuels rapports directs entre la tradition ésopique et l'aĩvos

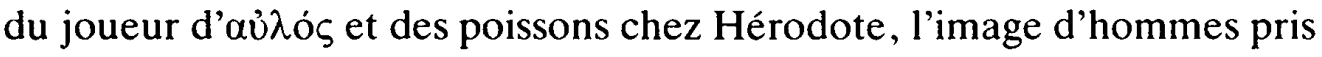
au filet comme des poissons est bien attestée dans la tradition grecque antérieure à Hérodote: on la trouve déjà dans l'Odyssée, où les prétendants tués par Ulysse sont comparés à des poissons: "Mais tous étaient couchés dans la boue et le sang; sous ses yeux, quelle foule! On aurait dit des poissons qu'en un creux de la rive les pêcheurs ont tirés de la mer écumante; aux mailles du filet, sur les sables, leur tas baille vers l'onde amère, et les feux du soleil leur enlèvent le souffle... C'est ainsi qu'en un tas gisaient les prétendants"20. L'image du filet qui va enserrer des soldats est utilisée par Sarpédon dans l'Iliade, lorsqu'il reproche à Hector de ne pas pousser ses soldats à la bataille: "Ah! J'ai peur que vous ne tombiez aux mailles d'un

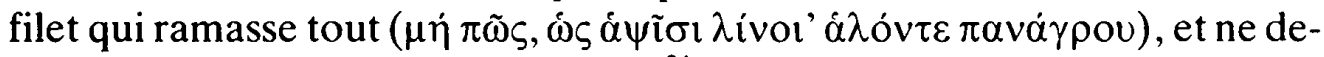
veniez la proie, le butin de l'ennemi"21. Quoiquc l'on n'y trouvc aucun des termes utilisés par Hérodote pour désigner le filet, ce passage est intéressant, car ce sont des Troyens qui vont être pris dans les mailles d'un filet; de plus, il s'agit probablement d'un filet de pêche, et Athénée a compris le passage dans ce sens: "Le fait qu'ils mangeaient (les Achéens à Troie) aussi du poisson est prouvé par Sarpédon, comparant la prise d'une ville à la pêche au grand filet" (Sophistes au banquet I, 25b). Le commentaire d'Athénée à propos des paroles de Sarpédon montre que la comparaison utilisée est largement connue; dans l'Agamemnon d'Eschyle, le chœur recourt à la même image pour décrire la prise de Troie: "O Zeus Roi, ô Nuit amie qui nous a conquis de telles splendeurs! Toi qui as jeté sur les murs de Troie un filet enveloppant, et ni enfant ni homme fait n'a pu s'évader du vaste réseau d'esclavage où le malheur les a tous faits prisonniers" 22 .

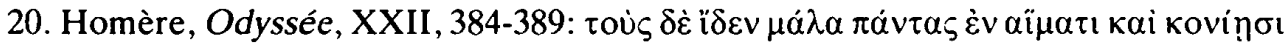

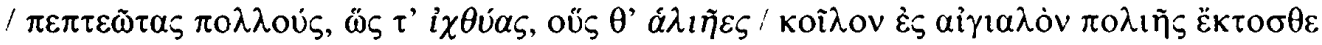

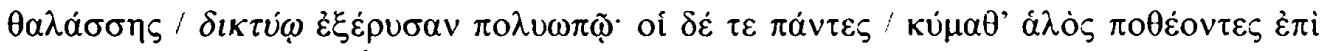

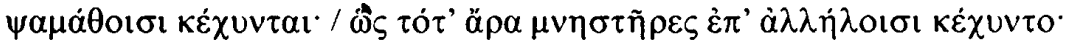

21. Homère, Iliade, V, 486.

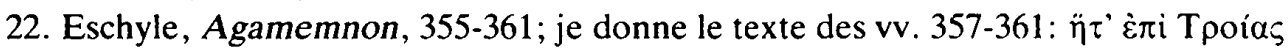

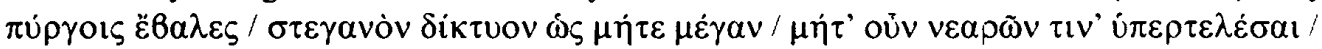

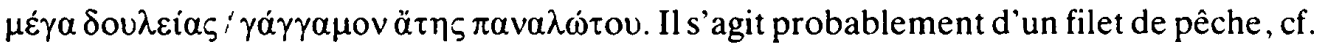
E. Fränkel, Aeschylus. Agamemnon, Oxford, 1950, II, pp. 189-190; contra, J. Dumor- 
L'image du filet auquel on ne peut échapper hante par ailleurs l'Agamem-

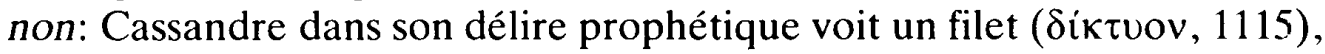
le filet d'Hadès, voile qui va enserrer Agamemnon; Clytemnestre, après avoir tué Agamemnon, dit avoir tendu autour de lui "un réseau sans issue,,

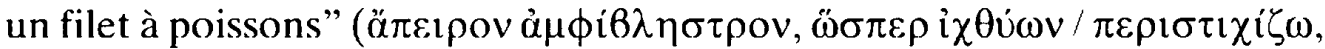
Aesch. Ag. 1382-83). Ce filet revient dans les Choéphores: Electre dit, en priant sur la tombe de son père: "Souviens-toi du filet de leurs ruses

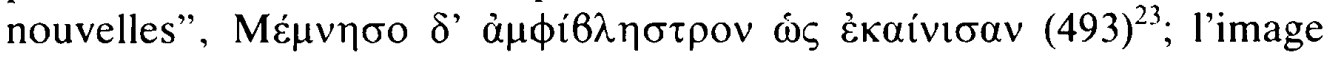
réapparaît, dans la bouche d'Oreste, pour rappeler le crime de Clytemnestre (998-1000). Il s'agit là d'un filet à mètis: en effet, si le filet représente souvent la nécessité à laquelle on ne peut échapper, il est aussi une arme de la ruse ${ }^{24}$.

Une distinction entre le filet en tant qu'arme de la ruse et le filet comme représentation d'une contrainte à laquelle il est impossible de se soustraire apparaît ici nécessaire. En effet, le filet du flûtiste-pêcheur de l'aĩvo Cyrus n'est pas connoté comme arme de la ruse ${ }^{25}$; la ruse est également absente de l'image utilisée par Sarpédon dans l'Iliade et par le chœur des Ar-

tier, Les images dans la poésie d'Eschyle, Paris²1975 [1935], pp. 71-87. Le filet d'ä $\rceil \eta$ apparaît encore en Eschyle, Prométhée 1078: les Océanides, en restant auprès de Pro-

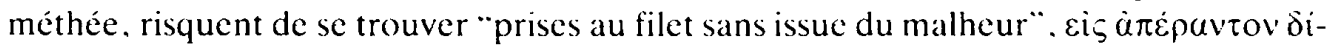

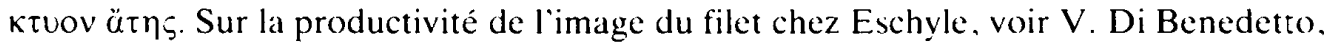
L ideologia del potere e la tragedia greca, Torino, 1978, pp. 3-4.

23. Oreste reprend le thème avec: "des chaînes sans airain où tu fus prisonnier" (494)

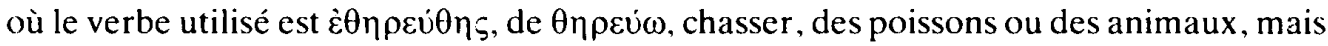

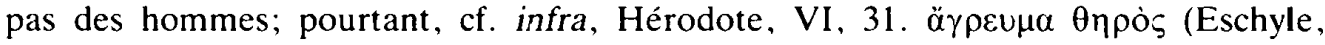
Choéphores, 998) est encore utilisé par Oreste pour connoter le filet qui donna la mort à Agamemnon.

24. Pour cette sorte de filet, voir M. Detienne et J.-P. Vernant, Les ruses de l'intelligence. La mètis des Grecs, Paris 1974: "le filet, invisible réseau de liens, est une des armes préferées de la mètis: c'est par le filet que Pittacos triomphe de Phrynon [Diog. Laert. I 74; Strabo XIII 600; Plut. De Her. Mal. 15], que Clytemnestre immobilise Agamemnon avant de l'égorger, qu'Héphaistos emprisonne Aphrodite et Arès [Od. VIII 278-280]. Le piège que tend Ulysse aux prétendants est un filet aux yeux innombrables [Od. XXII, 384-389, cf. supra]; les chaînes qui fixent Prométhée à son rocher tissent autour de lui un filet à mailles d'acier [Aesch. Prom. 81]", p. 51; et encore pp. 280282, où l'accent est mis sur le côté inéluctable de la prise au filet.

25. De même, La Fontaine, Fables, X, 10: "Les poissons et le berger qui joue de la flûte", interprète cet apologue dans le sens d'une opposition entre discours raisonnable et action brutale, comme l'illustre bien la morale de sa version de la fable: "O vous pasteurs d'humains et non pas de brebis, / Rois, qui croyez gagner par raison les esprits / D'une multitude étrangère, / Ce n'est jamais par là que l'on en vient à bout; / Il y faut autre manière: / servez-vous de vos rets, la puissance fait tout" (vv. 30-35). 
giens dans l'Agamemnon pour dépeindre la prise de Troie ${ }^{26}$. Dans tous ces cas, le filet symbolise l'impossibilité totale d'échapper, l'ànopía. Il est donc tout à fait possible de lire la fable du flûtiste et des poissons sur la base de ces images de cités prises au filet, ainsi qu'en rapport avec la comparaison de l'Odyssée entre les prétendants et des poissons pris au filet.

Il est encore une sorte de comparaison entre hommes et poissons qui peut être mise en relation avec notre texte, même si le filet en est absent: c'est celle qui utilise l'image de la pêche aux thons. Au chant X de l'Odyssée, les Lestrygons fracassent les vaisseaux des compagnons d'Ulysse avec des blocs gigantesques; "puis, ayant harponné mes gens comme des pois-

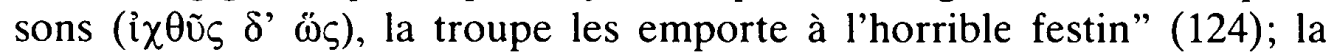
similitude est reprise par Eschyle dans la description, par le messager perse, de la bataille de Salamine: "les Grecs, comme s'il s'agissait de thons, de poissons vidés du filet ( frappent, assomment..." (Perses, 424-426). Hérodote, tout autant que son public, connaissait certainement ce texte célèbre; on peut remarquer que la comparaison est ici mise dans la bouche d'un Perse.

Plus importante encore est la présence chez Hérodote d'une telle comparaison dans un oracle qu'adresse à Pisistrate un devin grec d'Acarnanie, "homme qui s'y connaissait en prophéties" et qui était "mû par une inspiration divine" $(\theta \varepsilon i ́ n \pi o \mu \pi \tilde{n})$. Voici le texte de l'oracle: "Le filet est jeté, les rets sont déployés; pendant la nuit, à la lueur de la lune, les thons s'y précipiteront" "27. Suite à cet oracle, Pisistrate attaque les Athéniens au soir et s'empare d'Athènes pour la troisième fois. Le récit dont fait partie cet oracle est intégré à la narration des événements d'Asie Mineure; en effet, la digression sur l'histoire d'Athènes est motivée par le désir de Crésus de s'allier aux plus puissants parmi les Grecs (I, 56,1-2), et elle s'ouvre et se ferme par une référence annulaire à Crésus ${ }^{28}$. Cet oracle précède donc la réponse de Cyrus aux Ioniens, aussi bien du point de vue de la narration que du point de vue chronologique, puisqu'il est mis en rapport avec l'in-

26. Dans le cas d'Eschyle, c'est à partir de la première prise au filet d'une cité par Zeus et la Nuit, prise qui n'est pas connotée autrement que comme "nécessité", que se développe une isotopie du filet (cf. encore Eschyle, Agamemnon 868 et 1609-1611); l'image va basculer de plus en plus du côté de la ruse, jusqu'à redevenir neutre avec Choéphores 505-507: "Les enfants d'un héros sauvent son nom de la mort, ainsi que le

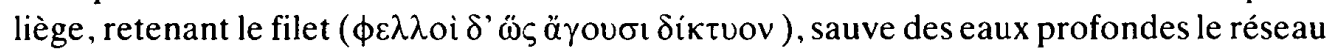
de lin".

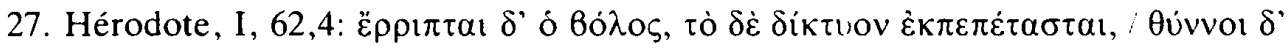

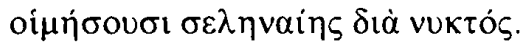

28. Hérodote, I, 59,1; Hérodote I 65, 1 . 
tervention de Crésus; mais l'image est ici utilisée par un Grec et adressée à un Grec, qui la comprend parfaitement ${ }^{29}$.

La réponse de Cyrus aux Ioniens peut être lue à l'intérieur d'un réseau d'allusions tout à fait grec; cela ne suffit pas pour exclure complètement la possibilité d'un référent perse, cela rend toutefois très probable l'hypothèse d'une manipulation d'Hérodote. A ce stade de notre analyse, nous pouvons affirmer que la réponse qu'Hérodote attribue à Cyrus réveille toutes sortes d'échos auprès d'un auditeur-lecteur grec; et qu'il est possible qu'Hérodote, pour des raisons encore à déterminer, ait voulu faire en sorte que Cyrus s'exprime par une fable grecque, renvoyant à une image très productive $\mathrm{au} \mathrm{V}^{\mathrm{e}}$ siècle.

\section{Parallèles dans la tradition orientale}

\subsection{Peuples conquis comme des poissons dans la tradition orientale.}

Le filet apparaît en tant qu'arme pour emprisonner des captifs déjà à l'époque sumérienne: la stèle des Vautours (première moitié du $\mathrm{III}^{\mathrm{e}}$ millénaire) représente un dieu mésopotamien capturant dans un filet les ennemis du roi ${ }^{30}$. Quant à la comparaison entre des peuples conquis et des poissons, si elle ne semble pas être attestée en Perse directement, elle est fréquemment utilisée par les rois assyriens. En effet, dans plusieurs inscriptions royales des $\mathrm{VIII}^{\mathrm{e}}$ et $\mathrm{VII}^{\mathrm{e}}$ siècles av. J.-C., les rois font allusion à des peuples "capturés comme des poissons". Sargon II proclame: "Je pêchai les Ioniens du milieu de la mer du soleil couchant comme si c'était du poisson et je déportai les gens de Kasku, Hilakku, Tabalu"31; le même

29. Sur le rapport entre tyran et filet, cf. encore C. Catenacci, "Il túpavvos e i suoi strumenti", Quaderni urbinati di cultura classica ns. 39, 3, 1991, pp. 85-95. Nous arrêtons ici la recherche d'autres parallèles dans le monde grec; les références que nous avons données suffisent à montrer que le thème est largement utilisé dans le monde grec à l'époque d'Hérodote.

30. Reproduction dans A. Parrot, Sumer, Paris 1981, n. 161; traduction de l'inscription accompagnant le relief in F. Sollberger et J.-R. Kupper, Inscriptions royales sumériennes et akkadiennes, Paris, 1971, pp. 47-55, IC 5a. Dans l'inscription, il est fait plusieurs fois mention d'un "filet de jet", qui doit s'abattre sur les transgresseurs des serments. On peut confronter ce texte avec l'Enouma Elish IV 41-44: Mardouk façonne un filet pour en entourer Tiamat, l'eau amère; 95 : il déploie son filet et l'en enveloppe; 112: les dieux alliés de Tiamat "furent jetés dans des filets, mis à demeure dans des rets". Mardouk fend ensuite le cadavre de Tiamat: "en deux il le fendit, comme un poisson séché", IV 137. Cf. encore V 64 et 71; VI 83 (in Textes babyloniens, ougaritiques, hittites, présentés et traduits par R. Labat et al., Paris 1970).

31. Khorsabad, Fastes de la salle XIV, cf. D. Luckenbill, Ancient Records of Assyria 
événement est mentionné dans l'inscription du taureau: “...Moi qui ai pêché les Ioniens du milieu de la mer comme des poissons" 32 . Une formulation un peu différente du même événement se trouve dans le prisme de $\mathrm{Nimrud}^{33}$, ainsi que, avec encore plus de détails, dans les quatres cylindres de Khorsabad: "[moi qui], puissant au combat, ai pêché les Ioniens du milieu de la mer à la manière d'un pêcheur (?), comme du poisson, et soumis Qué et Tyr" ${ }^{34}$. Nous trouvons ici la comparaison entre peuples maritimes vaincus et poissons, et la métaphore de la pêche, réunies en une seule formulation. A plusieurs reprises, des textes de Sargon II parlent d'un "roi de Dilmun qui se trouve à une distance de 30 beru au milieu de la mer, comme un poisson" 35 . Esarhaddon raconte en ces termes la suppression de la rébellion phénicienne (677-676 av. J-.C.): "Abdi-Milkutti, roi de Sidon, qui n'a pas eu crainte de moi, qui n'a pas écouté ma parole, qui a mis sa confiance dans la mer terrible et a secoué mon joug- Sidon sa cité fortifiée, qui se trouve au milieu de la mer, je l'ai terrassée comme un ouragan, j'ai arraché et jeté dans la mer ses murs et ses habitations; l'endroit où elle se trouvait, je l'ai rendu désert. Son roi Abdi-Milkutti, qui avait fui sur la mer devant mes armes, sur l'ordre de mon seigneur Assur comme un poisson je l'ai capturé et sorti hors de la mer et j'ai coupé sa tête".36. Plus

and Babylonia. II. Chicago, 1927. $§ 80$; texte tiré de la traduction de J. Elayi-A. Cavigneaux, "Sargon II et les Ioniens", Oriens Antiquus, 18, 1979, p. 70. Comme le fait remarquer Hirsch, art.cit., n. 5, il est particulièrement intéressant que la similitude avec des poissons s'applique aux Iamanéens, c'est à dire aux Ioniens ou Grecs.

32. A Khorsabad; Luckenbill II § 92, Elayi-Cavigneaux, art.cit., p. 71. Même texte dans le Pavé de Porte, toujours à Khorsabad, Luckenbill II $\$ 99$, Elayi-Cavigneaux, ibid.

33. Prisme ND 3411: "[moi] qui ai pêché les Ioniens du milieu de la mer à la manière d'un pêcheur (?)", cf. Elayi-Cavigneaux, art.cit., p. 71; texte chez J. Gadd, "Inscribed Prisms of Sargon II from Nimrud", Iraq 16, 1954, pp. 199-120, 1.19. Le mot sandânis, traduit par Elayi-Cavigneaux, selon la proposition de W. von Soden, Akkadisches Handwörterbuch, I-III, Wiesbaden 1958-1981, s.v. sandânis, "à la manière d'un pêcheur". est traduit par Gadd, ibid. "in a net", en un filet.

34. Elayi-Cavigneaux, art.cit., p. $71=$ Luckenbill II $\$ 118$.

35. Luckenbill II $\$ 41,43,70,81,99,160$. Cf. aussi, sous Esarhaddon, 1e $\$ 539$. Sur Sargon II, voir Cambridge Ancient History'2, III 2, 1991, pp. 88-102.

36. D'après la traduction en allemand de R. Borger, Die Inschriften Asarhaddons, Königs von Assyrien, Archiv für Orientforschung beiheft 9. Graz 1956, Nin. A II 65-74a (cf. aussi $\mathrm{A}^{5}$ et $\mathrm{A}^{16}$, Nin.D) = Luckenbill II $\$ 511$ (oủ le texte n'est pas complet); presque le même texte in Borger Nin. B I 10-19 (cf. aussi Nin. B ${ }^{3}$ ) = Luckenbill II § 527; cf. encore Borger AsBbE 2b-3a: "J'ai conquis [Sidon, qui se trouve au milieu de la mer; son roi Abdi-Milkutti] je l'ai sorti [de la mer] comme un poisson et je lui ai coupé la tête, = Luckenbill II § 710. Pour ces événements, voir Cambridge Ancient History ${ }^{2}$, III 2, 1991, pp. 124-126. 
significatif encore un autre épisode, toujours lié aux campagnes d'Esarhaddon: "Des rois, qui habitent dans la mer, dont les murailles sont la mer et le rempart les flots, qui empruntent comme voiture un navire et qui harnachent au lieu de chevaux des rameurs, eurent très peur (...). Qui pour se sauver avait fui au large dans la mer n'échappa pas à mon filet, je ne lui permis pas de s'enfuir (ou: il ne put sauver sa vie). Celui aux pieds rapides, qui avait monté vers les montagnes éloignées, je le tirai en bas hors de la montagne comme un oiseau (...). Aucun de ceux qui avaient fait de la mer leur refuge ou de la montagne leur soutien, ne glissa hors de mon filet; certainement, il n'y arriva pas. A l'habitant de la mer j'assignai d'habiter la montagne, au montagnard la mer" ${ }^{\text {37. }}$.

Ces textes sont très explicites, et s'agissant d'inscriptions royales, s'ils ne reflètent pas toujours la réalité, ils reflètent certainement l'idéologie des dominateurs assyriens. A ces inscriptions on peut encore ajouter des textes hébreux de l'Ancien Testament, en relation dans la majorité des cas avec le siège et la conquête de Jérusalem par les Néobabyloniens en 587. C'est ainsi que le prophète Habacuc, après avoir évoqué l'arrivée du conquérant chaldéen, lance un appel au Seigneur en ces termes: "Tu fais désormais les hommes à l'image des poissons dans la mer, de ce qui grouille sans maître: celui-là (scil. le conquérant) les tire tous à l'hameçon, il les drague au filet, les ramasse au chalut" ${ }^{138}$. On peut situer ce texte vers la fin du VII ${ }^{\mathrm{e}}$ sièvle av. J.-C. Vers la même époque, Jérémie annonce ainsi la proche destruction de Jérusalem: "Je vais envoyer quantité de pêcheurs oracle du Seigneur - qui les pêcheront; et puis jenverrai quantité de chasseurs qui les chasseront sur toute montagne, sur toute colline et jusque dans les creux des rochers. Mon regard est braqué sur toutes leurs démarches, rien ne m'échappe" $(J r .16,16-17)$. Dans ces deux cas, ce sont des hommes, les conquérants, qui se servent du filet comme d'une arme pour pêcher des hommes; mais le filet est aussi l'arme du Seigneur, notamment chez Ézéchiel qui, à propos de la capture du roi de Jérusalem Sédécias (en 587 av. J.-C.), rapporte les paroles du Seigneur: "J'étendrai mon filet sur lui et il sera pris dans mes rets" ${ }^{\prime 39}$. Des poissons apparaissent

37. Borger Nin. A IV 82-85; V 10-13a; 17-20.

38. Habacuc 1, 15; cf. aussi Habacuc 1, 16-17. Les traductions de textes de l'Ancien Testament sont tirées de La Bible, traduction œcuménique, Paris 1988. Sur les diverses utilisations du filet dans la Bible, voir F. Vigouroux, Dictionnaire de la Bible, Paris 1912. s.v. "Filet", et plus récemment W.H. Wuellner, The Meaning of "Fishers of Men", Philadelphia 1967.

39. Ezéchiel 12, 13; la phrase est reprise, toujours à propos du même Sédécias, un peu plus loin $(17,20)$. 
dans l'oracle du Seigneur dirigé, dans le même contexte, contre le Pharaon égyptien: "Je viens contre toi, Pharaon, roi d'Égypte, grand dragon tapi au milieu de ses Nils... Je mettrai des crochets à tes mâchoires, j'attacherai les poissons de tes Nils à tes écailles, je te tirerai du milieu de tes Nils avec tous les poissons de tes Nils attachés à tes écailles. Je te jetterai dans le désert avec tous les poissons de tes Nils" ${ }^{\text {"40 }}$. C'est d'ailleurs à cette tradition que fait référence la métaphore du "pêcheur d'hommes" dans les Évangiles" La capture de poissons au filet comme métaphore de la capture d'hommes est donc très répandue dans tout le Proche-Orient; nous l'avons vue déjà, avec la Stèle des Vautours, à une période très ancienne; l'Enouma Elish, vers le $\mathrm{XI}^{\mathrm{e}}$ siècle, atteste la continuité de cette représentation; les textes des prophètes enfin couvrent la période comprise entre les inscriptions assyriennes et l'avènement de Cyrus.

Hirsch a donc sans doute raison d'affirmer que le recours à la comparaison avec des poissons reflète l'attitude naturelle d'un pouvoir continental, comme l'Assyrie, envers des peuples qui habitent près de la côte ${ }^{42}$. N'ayant pas trouvé de parallèles perses, il est obligé de supposer qu'il est fort probable, au vu de l'influence exercée par l'Assyrie sur l'empire perse et du fait que ce dernier est aussi un pouvoir continental, que les Perses et Cyrus aient partagé une conception propre aux Assyriens, et que, par conséquent, les Perses aussi aient employé pour se représenter les habitants des côtes et des îles l'image de poissons vivant dans la mer. Nous voyons maintenant qu'il n'est pas possible de parler d'une conception propre aux Assyriens: il s'agit plutôt d'une représentation assez répandue dans l'Orient ancien. L'apologue de Cyrus dans les Histoires serait donc à comprendre en rapport avec cette représentation.

\subsection{Eléments tirés de la tradition grecque}

En fait, on peut apporter d'autres éléments, internes aussi bien qu'ex-

40. Ézéchiel 29, 3-5; cf. Ézéchiel 32, 3. Voir encore, pour le filet comme arme, Job 18 , 7-9; 19, 6; 22,10; et Psaumes 35, 7-8; 140, 6.

41. Matthieu 4, 18-20; Marc 1, 16-19, Luc 4, 5-11. Cf. aussi la parabole de Matthieu 13. 47-48.

42. "Presumably the conquest of peoples living on islands or in well fortified towns posed special problems for the land-based Assyrian military machine, and the Assyrians might well regard the capture of such places as akin to the achievement of a land-bound fisherman who manages to draw in fish from the sea with his net", art.cit., 224. On peut confronter cela avec une inscription de Ninive relatant les conquêtes d'Esarhaddon (Borger Nin. A III 20-31 = Luckenbill II $\S 513$; cf. pour une version proche $\S 528$ et $\S$ 521), qui montre clairement que la situation géographique se reflète sur la caractérisation de l'habitant. 
ternes au texte d'Hérodote, pour montrer que l'image de poissons pris au filet pour dépeindre la soumission de populations côtières n'est pas étrangère aux Perses.

En effet, à deux reprises Hérodote fait allusion à une technique appliquée par les Perses au moment de la conquête d'une île. En III 149, Hérodote nous dit que les Perses prirent Samos au filet ( $\tau \hat{\eta} \nu \delta \dot{\varepsilon} \Sigma \dot{\alpha} \mu \mathrm{ov}$

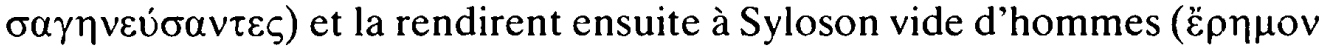
غ̇oṽ $\sigma \alpha v \dot{\alpha} v \delta \rho \tilde{\omega} v$; nous sommes aux alentours de 520 av. J.-C.). Hérodote ne ressent manifestement pas le besoin d'expliquer en quoi consiste cette technique; il se borne à une allusion rapide, ce qui montre que ses auditeurs devaient savoir suffisamment bien de quoi il s'agissait. La seconde mention d"une "prise au filet" de populations insulaires intervient en VI 31 . 1-2, après la défaite de la flotte ionienne à Ladé et la prise de Milet, en 493 av. J.-C. Cette fois, Hérodote décrit de façon détaillée ${ }^{+3}$ la façon de procéder des Perses:

L'armée navale perse... s'empara facilement des îles voisines du continent, Chios, Lesbos et Ténédos. Chaque fois qu'elle en prenait une, les Barbares en s'en emparant y capturaient les habitants comme dans un filet

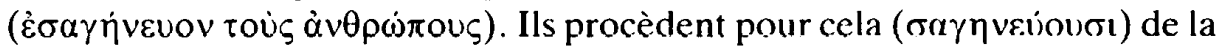
façon suivante. Les soldats, se tenant par la main, forment une chaîne qui va du rivage Nord au rivage Sud; ils avancent ensuite à travers l'île entière et

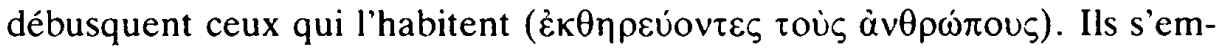
parèrent aussi dans les mêmes conditions des villes continentales de l'Ionie, à cela près qu'ils n'en capturèrent pas les habitants comme il vient d'être dit

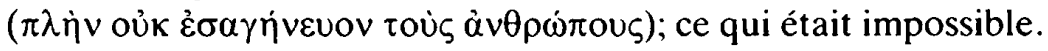

Dans l'œuvre d'Hérodote, une telle pratique n'est attribuée à aucun autre peuple; elle est typique des Perses. Les commentateurs modernes ont discuté de la possibilité réelle de son application: il a semblé impossible que la chaîne de soldats ait pu tenir en face des obstacles naturels (vallons, torrents, forêts) que présentent les îles ${ }^{44}$. Mais contre ces doutes on peut faire valoir qu'Hérodote lui-même est conscient de la difficulté technique d'un tel procédé, puisqu'il dit qu'il n'a pas été possible de l'appliquer pour les cités sises sur le continent asiatique; s'il accepte donc la tradition du

43. On peut expliquer le fait qu'il ne donne des détails que la seconde fois par sa volonté de souligner émotivement la "fin en catastrophe" de la révolte d'Ionie; pour une autre raison, cf. infra $\mathrm{n} .55$.

44. Cf. les hésitations de W.W. How \& J. Wells, A Commentary on Herodotus, I-II Oxford 1912, II p. 74: "In fact, this 'netting' of the population would be as impossible in the mountains and clefts of the smaller islands as in Eubœa". 
"ratissage" des îles, c'est après l'avoir soumise à un examen critique. D'ailleurs, en dehors du texte d'Hérodote, nous avons des parallèles pour un tel procédé: on peut ajouter aux textes d'Esarhaddon déjà mentionnés une inscription assyrienne du règne d'Assurbanipal: "[Dans ma troisième campagne] contre Ba'li, roi de Tyr, [qui vit dans le milieu de la mer] je marchai. Puisqu'il n'écoutait pas mes ordres royaux (...) je tendis un cordon puissant, d'où on ne pouvait s'échapper, autour d'eux" ${ }^{45}$. Enfin, dans un article de 1954, Karl Meuli réunissait des témoignages ayant trait à la "Treibjagd" chez les Mongols ${ }^{+10}$. Un trait typique de cette chasse est d'être en même temps un entraînement militaire: la Cyropédie nous présente un exemple d'une de ces grandes chasses où les animaux étaient rabattus par l'armée, chasse qui tout à coup tourne à l'expédition militaire ${ }^{47}$. Dans cette optique, il faudrait valoriser l'utilisation qu'Hérodote fait du verbe

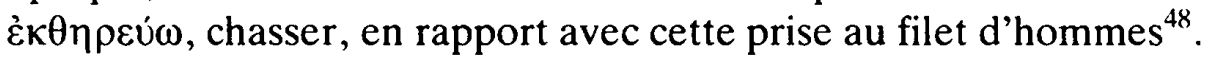

Ce procédé perse frappa énormément les Grecs. Cela apparaît entre autres de la mention que Platon en fait dans le Ménexène et dans les Lois $^{49}$ : au cours de la première Guerre médique, les Perses auraient passé au filet

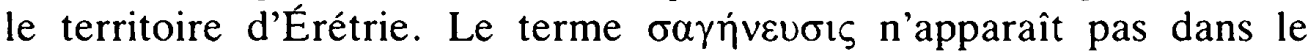
Ménexène, mais la description de l'opération est très proche de celle donnée par Hérodote à propos des îles ioniennes et éoliennes, Chios Ténédos et Lesbos (VI 31,2). En revanche, le Ménexène s'écarte de la narration de la prise d'Érétrie par Hérodote (VI 101) sur deux points: à propos de la durée du siège (trois jours au lieu de sept), et en appliquant à Érétrie le schéma de la $\sigma \alpha \gamma \dot{v} v \varepsilon v \sigma ı$ :

45. Luckenbill II $\$ 847$. La comparaison avec les poissons pour indiquer des peuples qui vivent au bord de la mer est attestée aussi sous le règne d'Assurbanipal (668-626), cf. Luckenbill II $\$ 912$.

46. K. Meuli, "Ein altpersischer Kriegsbrauch", in Westöstliche Abhandlungen Rudolf Tschudi zum siebzigsten Geburtstag, Wiesbaden 1954, repris in Gesammelte Schriften, Basel-Stuttgart 1975, II, pp. 699-729; Meuli proposait (p. 723) de reconnaître une "Treibjagd" dans les reliefs en albâtre ornant une salle du palais d'Assurbanipal à Ninive.

47. Xénophon, Cyropédie, I 4, 16-17, et Meuli, art.cit., pp. 723-724.

48. Cf. aussi Eschyle, Choéphores, 494 et 998, cit. supra n. 23.

49. Platon, Ménćxène, $240 \mathrm{~b}$, "passage au filet" de la $\chi \omega \dot{\rho} \rho \alpha$ d'Érétrie par l'armée perse: Lois, III. 698d, Datis fait savoir à Athènes qu il a "ratissé" au filet Érétrie. Le débarquement en trois points différents de l'île (l'Eubée, mème si elle est grande, est quand-même une île; cela est important pour la représentation grecque de ce "passage au filet"). raconté en Hérodote VI 101. l et la déportation de la population auraient rendu un tel procédé plausible. 
Datis, ayant fait voile vers Érétrie contre des hommes qui étaient alors en Grèce parmi les plus réputés dans l'art de la guerre, et qui se trouvaient en nombre, les soumit en trois jours et fouilla tout leur pays, pour n'en laisser échapper aucun, de la manière suivante: arrivés aux frontières d'Érétrie, ses soldats firent la chaîne d'une mer à l'autre en se tenant par la main, et traversèrent ainsi tout le territoire pour pouvoir dire au Grand Roi que nul ne leur avait échappést.

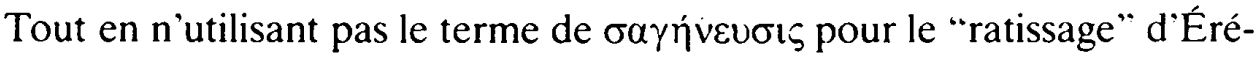
trie. le Ménexène renvoie donc de très près à la description donnée par Hérodote de ce procédé. Le terme $\sigma \alpha \gamma \eta \dot{v \varepsilon v \sigma ı s ~ a p p a r a i ̂ t ~ e n ~ r e v a n c h e ~ d a n s ~}$ les Lois. Platon ne s'intéresse pas ici à la réalité effective de cette 'prise au filet' des Érétriens ${ }^{51}$; ce qui retient son attention, c'est le choc provoqué par cette nouvelle sur les Athéniens et sur les Grecs en général; on peut le croire quand il affirme que le choc fut énorme. En 490 av. J.-C. donc, les Athéniens ne se demandèrent pas dans quelle mesure un tel procédé, qui devait certainement leur être connu par les récits des rescapés de la révolte d'Ionie, était applicable.

Si l'on admet l'existence d'un référent historique pour le procédé perse de la $\sigma \alpha \gamma \eta \dot{v \varepsilon v \sigma ı s, ~ i l ~ a p p a r a i ̂ t ~ t o u t ~ a ̀ ~ f a i t ~ p o s s i b l e ~ d ' a d m e t t r e ~ a u s s i ~ l ' e x i s-~}$ tence d'un tel référent pour l'apologue du joueur de flûte et des poissons. La comparaison avec les inscriptions assyriennes montre que Cyrus a certainement pu répondre aux Ioniens en des termes très proches de ceux qui nous sont rapportés par Hérodote.

Nous avons jusqu'à présent éprouvé les possibilités d'une démarche visant à définir l'historicité de l'anecdote au moyen de la recherche de parallèles externes au texte. Mais cette démarche nous a conduit, en quelque sorte, vers une impasse: nous avons trouvé à la réponse de Cyrus des paral-

50. Il y a des correspondances très marquées entre le Ménexène et Hérodote, VI, 31,2

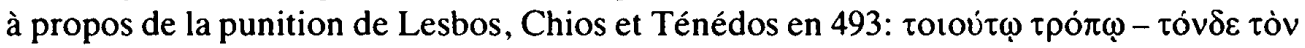

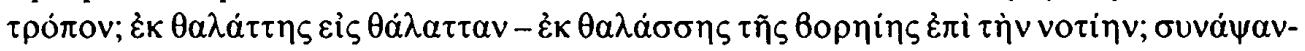

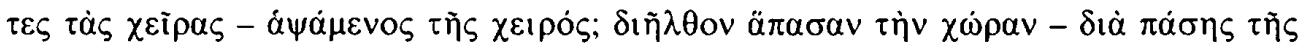

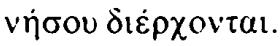

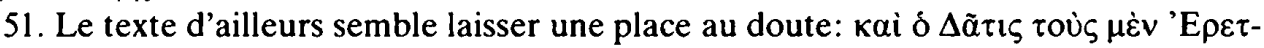

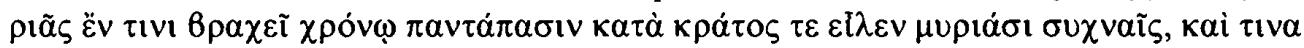

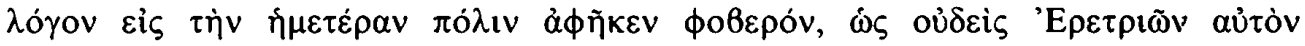

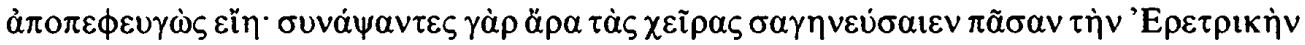

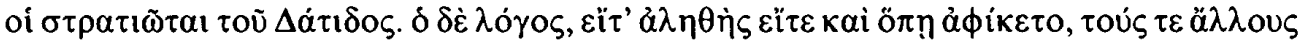

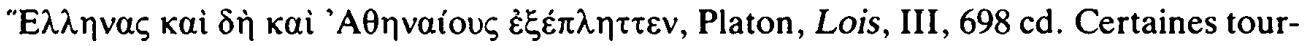

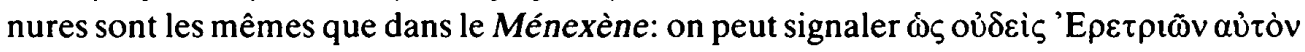

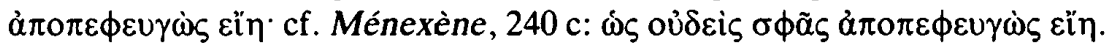


lèles très proches aussi bien dans la tradition orientale que dans la tradition grecque; nous ne pouvons donc pas trancher la question de l'historicité de l'anecdote. Tout ce que les parallèles orientaux nous permettent d'affirmer, c'est d'une part qu'il est fort probable que Cyrus ait réellement employé ces mots, et d'autre part que le choix d'Hćrodote d'accepter cette tradition peut se justifier. Quant aux parallèles dans la tradition grecque, ils nous ont du moins permis de délimiter l'horizon d'attente esthétique des destinataires: nous avons pu en effet mettre en rapport la structure formelle de cette anecdote avec la fable ésopique et avec l'aĩvos; d'autre part, nous avons vu que sur le plan sémantique la réponse de Cyrus renvoie à une comparaison entre hommes et poissons pris au filet ${ }^{52}$, bien connue dans la tradition grecque.

Nous ne savons cependant toujours pas ce qui a guidé le choix d'Hérodote et ce qui l'a poussé à intégrer cette fable à son texte. Nous allons maintenant procéder à une analyse interne au texte d'Hérodote; c'est la seule qui puisse nous permettre d'apporter une réponse à la deuxième question; peut-être pourra-t-elle nous donner également des indications quant à la question de l'historicité de l'anecdote.

\section{Analyse du texte d'Hérodote}

\subsection{Le rapport entre l' $\alpha \tilde{l} v o \varsigma$ de Cyrus et la $\sigma \alpha \gamma \tilde{v} v \varepsilon v \sigma ı \varsigma$}

Platon semble accepter sur le siège d'Érétrie une tradition différente de celle rapportée par Hérodote; en même temps, Platon (ou la tradition que suit le philosophe) applique implicitement ou explicitement au siège

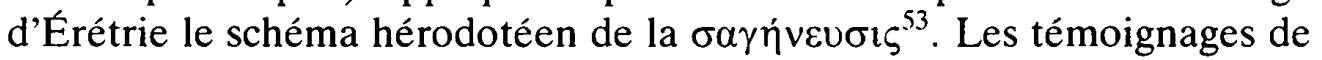
Platon sur la prise au filet de la population d'Érétrie nous poussent à examiner de plus près les choix d'Hérodote quant à ces "prises au fillet”. En effet, comme nous l'avons dit, pour Érétrie Hérodote ne parle pas de $\sigma \alpha \gamma \eta \dot{v \varepsilon v \sigma ı s . ~ P e u t-e ̂ t r e ~ c e ~ s i l e n c e ~ e s t-i l ~ d u ̂ ~ t o u t ~ s i m p l e m e n t ~ a u ~ f a i t ~ q u e ~ l e s ~}$ Perses ne ratissèrent pas le territoire d'Érétrie. Si l'on regarde de près les passages hérodotéens relatifs à la prise d'Érétrie, on s'aperçoit que VI

52. Cf. Payen, art.cit., p. 530, à propos de la narration du règne de Cambyse: "Hérodote travaille donc sur un double fond de tradition esthétique qui, entrant dans le champ des connaissances de ses lecteurs-auditeurs, facilite la mémorisation et la compréhension de son cuvre".

53. F. Lasserre, Strabon, Géographie X, Paris 1971, note compl. p. 119, pense que le récit des Lois dériverait d'Éphore, qui aurait introduit le terme $\sigma \alpha \gamma \eta \dot{v \varepsilon v \sigma \iota \varsigma ~ c o m m e ~ u n ~}$ "hérodotisme", tandis que la version du Ménexène, ne comportant pas cette expression, dépendrait d'Hellanicos. 
101,2 semble effectivement faire référence à un dépeuplement total: tous les hommes auraient été pris, ce qui correspond aux instructions de Darius à Artaphernès et Datis (VI 94,2: la cité, donc l'ensemble des citoyens, doit être réduite en esclavage). Cependant déjà en VI 119,1 apparaît un partitif ("ceux des Érétriens qui avaient été réduits en esclavagc"54), et des Érétriens participent aussi bien à la bataille de Salamine qu'à celle de Platée. On ne peut en définitive pas exclure que le silence d'Hérodote ne soit dû à des raisons inhérentes à la réalité historique des faits, donc à des raisons externes au texte. Cependant, il est aussi légitime de se demander si ce silence ne pourrait être dû au fait que l'Eubée se trouve en Grèce, en

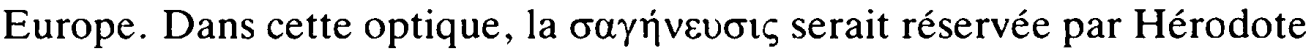
exclusivement aux îles de l'Ionie et de l'Éolide, et plus généralement aux populations maritimes de l'Asie Mineure ${ }^{55}$. En effet, Hérodote se sent dans l'obligation de faire une remarque à propos de la $\sigma \alpha \gamma \eta \dot{v} v v \sigma ı \varsigma$ manquée des cités continentales d'Asie Mineure, ce qu'il ne fait ni pour Érétrie ni pour Naxos (VI 96). Pourtant Naxos est aussi une île; c'était d'ailleurs, nous dit Hérodote, le premier but des Perses, puisque déjà avant la révolte d'Ionie ils avaient essayé de la conquérir (VI 95,2-96,1); l'île est liée d'une certaine façon aux événements de la révolte, même si elle n'y a pas participé en envoyant des contingents; elle n'est toutefois pas punie de la même façon que les insulaires ioniens et éoliens. Ce silence peut s'expliquer par le fait que Naxos est une des Cyclades: nous sommes en dehors des limites touchées par l'aĩvos de Cyrus.

Déjà Meuli, dans la conclusion de son article, avait mis en évidence le rapport existant entre la technique guerrière d'encerclement des ennemis par l'armée, donc de prise au filet orientale, et l'ainos de Cyrus; il en concluait à l'historicité probable des mots de Cyrus ${ }^{56}$. Ni Meuli ni Hirsch, qui

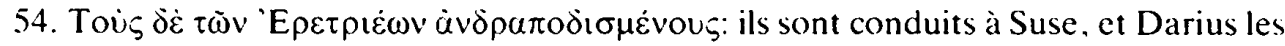
établit en Cissie, VI, 119,2.

55. Les Samiens font aussi partie des gens concernés par l’ainos, ce qui “justifie" que le procédé de la $\sigma \alpha \gamma$ ñvevoıs soit appliqué contre eux (Hérodote, III, 149, cit. supra); on peut aussi expliquer le fait qu'Hérodote choisit de donner des détails sur ce procédé

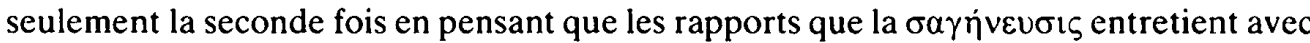
l'aivos de Cyrus poussent à souligner les cas où elle est appliquée aux Grecs d'Asie Mineure en bloc.

56. "Ich möchte glauben, Herodot habe uns hier ein echtes, geschichtliches Wort des Kyros bewahrt. Dass es überhaupt bewahrt und herumgeboten wurde, ist wohl verständlich; nicht weniger, dass sich Kyros bildlicher Rede bediente, und nach der Vorhergehenden ebensowenig, dass er sich gerade dieses Bildes bediente", art. cit., p. 720. Pour l'idée opposée, W. Aly, Volkmärchen, Sage und Novelle bei Herodot und seine Zeitgenossen, Göttingen 1921, p. 53, et Nagy, op. cit., supra n. 7. 
pourtant renvoient tous deux à la Vie d'Apollonios de Tyane de Philostrate - Hirsch à propos des poissons dansants, Meuli à propos de la

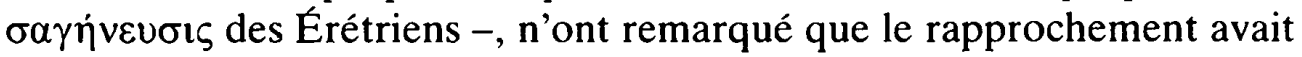
été fait dès l'antiquité, justement par Philostrate. En effet, dans le chapitre 23 du premier livre de la Vie d'Apollonios, Philostrate raconte qu'Apollonios, en passant par la Cissie, près de Babylone, voit en rêve des poissons qui, tombés de la mer sur la terre, se tortillent, en se plaignant d'avoir quitté leur élément ( $\tilde{\eta} \theta$ os). Il raconte le rêve à son compagnon Damis qui, effrayé, le supplie de ne pas aller plus loin: "afin d'éviter que nous-mêmes, comme les poissons, tirés hors de notre élément, ne périssions... ou ne soyons obligés de supplier quelque dynaste ou roi qui se moquera de nous". Cette première partie rappelle d'assez près l'anecdote des poissons dansants; l'explication du rêve par Apollonios, elle, est liée explicitement à la supposée ${ }^{57} \sigma \alpha \gamma \eta \dot{v \varepsilon v \sigma ı \zeta ~ d e s ~ E ́ r e ́ t r i e n s: ~ " E n ~ e f f e t ~ d e s ~ E ́ r e ́ t r i e n s ~ h a b i t e n t ~}$ cette terre de Cissie, déportés ici de l'Eubée par Darius il y a cinq cents ans, et on dit qu'ils furent traités, au moment de leur prise, comme les poissons que la vision a montré; en effet ils furent pris au filet ( $\sigma \alpha \gamma \eta v \varepsilon v \theta \tilde{\eta} v \alpha \iota)$ et tous capturés".

Ce texte montre clairement que l' $\alpha$ ĩvo des poissons dansants et en tout cas l'image de peuples conquis comme des poissons ont été mis en relation dès l'antiquité avec le procédé typiquement perse de la $\sigma \alpha \gamma \eta \dot{v} v \varepsilon \sigma ı \varsigma^{58}$. Et si chez Hérodote la $\sigma a \gamma \eta \dot{v \varepsilon v \sigma ı \zeta ~ n ' e s t ~ a p p l i q u e ́ e ~(o u ~ s o n ~ a b s e n c e ~ j u s t i f i e ́ e) ~}$ qu'aux Ioniens et aux Éoliens d'Asie Mineure, cela n'est peut-être pas sans rapport avec le fait que c'est précisément à ce groupe que Cyrus avait adressé son aĩvos. Il y a apparemment dans l'utilisation du thème de la "capture au filet" une cohérence interne très forte, que l'erreur de Strabon, at-

57. Cf. Meuli, art. cit., p. 702 n. 5: “Die zahlreichen späteren Erwähnungen des Netztreibens auf Eretria besagen natürlich nichts; sie gehen alle auf Plato zurück... Die

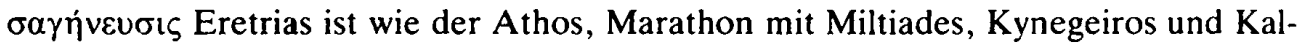
limachos ein Lieblingstopos der späteren Lobredner; vgl. z.B. Polemon Decl. I 30 p. 11,22; II 56, p. 35 Hinck; Max. Tyr. 23, 6e; Iulian. Or. II 79b Hertlein; Himer. Or. II 19; Phìostr. Vita Apoll. I 23, p. 14, $10 \mathrm{~K}$., um nur Stellen zu nennen, wo das Wort

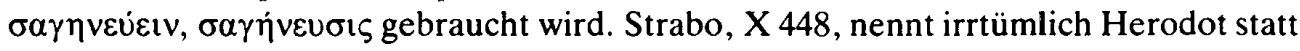
Plato als Gewährsmann".

58. Je rappelle que Grosso a montré, par d'autres arguments, que si Ctésias est probablement la source de Philostrate pour I 24, I 23 dépend en revanche d'Hérodote, cf. supra n. 15. Voir encore, même si elles sont moins significatives, les épigrammes sur le sort des malheureux Érétriens attribuées à Platon (Diogène Laërce III 33, Anthologie Palatine, VII, 256 et 259); dans tous ces textes, on joue sur l'image des marins transplantés au milieu des étendues continentales. 
tribuant à Hérodote le renseignement sur la $\sigma \alpha \gamma \eta \dot{v \varepsilon v \sigma ı \varsigma ~ d e s ~ E ́ r e ́ t r i e n s ~}{ }^{59}$, ne fait que rendre plus apparente. Si l'encerclement et la "prise au filet" décrits par Meuli s'appliquent à la conquête aussi bien de peuples insulaires que de peuples continentaux ${ }^{60}$, la désignation "poissons", elle, ne peut s'appliquer qu'à des peuples maritimes; d'ailleurs Hérodote dit que seuls les insulaires, parmi les Ioniens, furent "pris au filet", en soulignant une fois de plus le caractère maritime des peuples capturés au filet.

\subsection{La deuxième partie de l'ainos et la fin des Histoires}

Jusque là, nous n’avons pris en considération qu'une partie de l'uivos. Mais, s'il y a une correspondance claire entre la $\sigma \alpha \gamma \eta \dot{v \varepsilon v \sigma ı s, ~ " p r i s e ~ a u ~}$ filet" de "poissons", et la première partie de l'aìvo s de Cyrus, qu'en est-il de la deuxième partie de la fable, celle où les poissons dansent?

Il y dans les Histoires un répondant à cette deuxième partie de la fable; je crois qu'il est possible de montrer que l'anecdote des "poissons dansants", en plus de la fonction narrative de relater la réponse de Cyrus, joue un rôle important dans la structure des Histoires.

Nous retrouvons en effet des poissons dansants à la fin de l'œuvre. Après la bataille de Mycale, les Grecs se rendent à Abydos, afin de rompre les ponts que Xerxès avait jetés sur l'Hellespont. Lorsqu'ils y arrivent, ils trouvent les ponts déjà rompus ${ }^{61}$; la division entre l'Europe et l'Asie a donc été rétablie. A ce moment, les Athéniens passent à Sestos, en Chersonèse (donc en Europe), pour mettre le siège à la ville. C'est là en effet que se sont réunis les Perses restés en Europe, c'est là aussi que le commandant perse Oiobazos a fait apporter les cordages provenant des ponts. Le gouverneur de Sestos est Artayctès; de lui, Hérodote raconte que, homme dur et impie, il s'était approprié, en trompant Xerxès, des trésors de Protésilas, qui se trouvaient dans le sanctuaire d'Éléonte ${ }^{62}$. Après un siège très pénible, les Athéniens réussissent à s'emparer de la ville; ils capturent Artayctès, qui avait essayé de s'enfuir, et le gardent un certain temps prisonnier. C'est à ce moment-là des Histoires qu'interviennent à nouveau des poissons dansants:

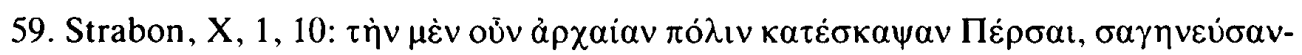

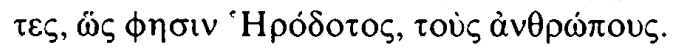

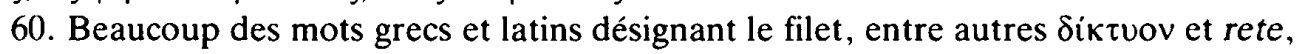
sont utilisés pour le filet de pêche comme pour celui de chasse et renvoient donc à des activités maritimes aussi bien que terrestres; voir à ce propos O. Longo, Le forme della predazione. Cacciatori e pescatori della Grecia antica, Napoli 1989, en part. pp. 21-37.

61. Hérodote, IX, 106; et IX 114.

62. Hérodote, IX, 116. 
D'après ce qu'on raconte en Chersonèse, il arriva qu'un des hommes qui les gardaient [Artayctès et son fils], pendant qu'il faisait cuire des poissons

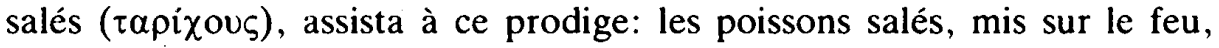
sautaient et palpitaient comme des poissons tout fraîchement pêchés (oi

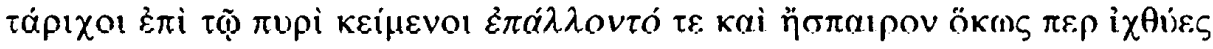
$v \varepsilon o \alpha ́ \lambda \omega \tau o l)$. Ceux qui faisaient cercle autour de lui étaient surpris; mais Artayctès, témoin du prodige, appela l'homme qui faisait cuire les poissons et lui dit: "Etranger d'Athènes, ne t'alarme point de ce prodige; ce n'est pas à toi qu'il s'adresse; c'est à moi que Protésilas d'Éléonte, bien que mort et salé

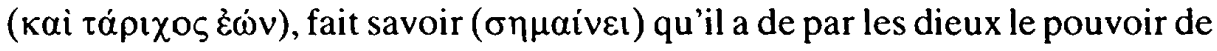
tirer vengeance des injures..." (Hérodote, IX 120).

Suite à cette interprétation du prodige, Artayctès propose un accord qui sera refusé par les Athéniens; conduit à l'endroit même où avait abouti le pont jeté par Xerxès d'une rive à l'autre du détroit, il y est crucifié, cloué sur des planches. Les Athéniens rentrent en Grèce, en emportant avec eux les câbles des ponts pour les consacrer dans leurs sanctuaires; l'ordre, avec la séparation des deux continents, est rétabli; et les Histoires se terminent sur un $\alpha \tilde{i} v o \zeta$, introduit par le nom d'Artayctès ${ }^{63}$, et dans lequel le rôle principal est joué encore une fois par Cyrus.

Aux deux moments où l'équilibre en Asie Mineure change, le même personnage, Cyrus, intervient avec un $\alpha \bar{i} v o \zeta$; le roi qui a refusé de s'accorder avec les Ioniens et qui a rattaché, pour la première fois, les insulaires à l'empire continental perse affirme, à la fin de l'œuvre, qu'il vaut mieux pour les Perses rester sur leur propre territoire et être indépendants, en commandant sur d'autres, plutôt que d'essayer de conquérir d'autres territoires, plus beaux et doués d'un climat plus favorable, pour être à la fin dominés. Aux paroles de Cyrus déconseillant aux Perses d'essayer de s'approprier un territoire riche, fertile, doté d'un bon climat, mais amollissant (IX 122), paroles qui suivent immédiatement le récit de la fin d'Artayctès, correspondent, dans le premier livre, les chapitres géo-ethnographiques sur les Ioniens et les Éoliens, qui suivent le refus de Cyrus, exprimé au

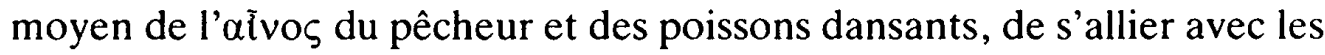
Grecs; ces chapitres s'ouvrent ainsi: "Les Ioniens dont il s'agit ici, ceux à qui appartient le Panionion, sont de tous les hommes que nous connais-

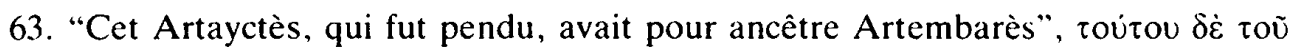
'A généalogie permet de remonter dans le temps tout en gardant un "ancrage" dans la situation contemporaine. 
sions ceux qui ont établi leurs villes sous le plus beau ciel et le plus beau climat" $"$.

S'il faut reconnaître que d'un point de vue terminologique la coïncidence entre la fable du premier livre et la résurrection des poissons au moment où Artayctès va mourir n'est pas très marquée ${ }^{65}$, en revanche ces deux épisodes s'insèrent dans une structure qui présente des parallélismes remarquables. Il est possible de représenter la relation entre les deux passages selon le schéma suivant:

Cyrus

alvos(I 141) - beau territoire (I 142,1) | poissons (IX 120)- aivos(IX 122)

poissons beau territoire

Le nom d'Artayctès qui introduit le dernier aivo $\varsigma$ des Histoires nous autorise à considérer les ch. IX 120-122 comme un seul bloc narratif. Dans le premier comme dans le dernier livre donc, Cyrus adresse un $\alpha$ โ̇vo, la première fois aux Grecs, la seconde aux Perses. Aux poissons fraîchement pêchés (métaphore des Grecs d'Asie Mineure) de l'aĩvo pondent dans le récit du narrateur les poissons secs ressuscités (métaphore de Protésilas mais aussi, nous le verrons, des Grecs d'Asie Mineure). Enfin, à la remarque du narrateur sur le beau territoire occupé par les Ioniens, remarque qui suit l' $\alpha$ Ĩvo $\zeta$ de Cyrus dans le premier livre, correspond le contenu de l'aîvo

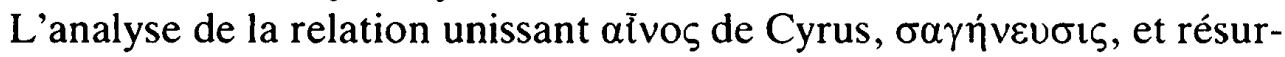
rection des poissons à la fin des Histoires, conduit à des résultats intéressants.

En effet, nous avons, avec la soumission des îles à Cyrus (soumission qui suit de peu sa conquête des cités maritimes d'Ionie ${ }^{66}$ ), une rupture historique, liée à l'arrivée des Perses (après les ruptures mythiques liées aux rapts de femmes), de l'équilibre naturel Europe-Asie: les poissons sont

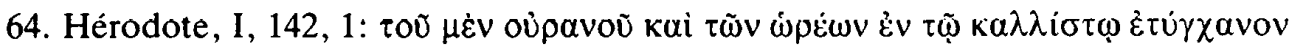

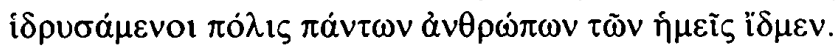

65. On peut signaler l'utilisation du même verbe, $\pi \dot{\alpha} \lambda \lambda \varepsilon \iota v$, pour indiquer les sauts des poissons. Il est difficile d'imaginer que l'auditeur/lecteur aurait été attentif, à une telle distance, à des rapports terminologiques; en revanche, le rapport structurel plus général aurait pu être perçu. Au-delà du problème de la perception éventuelle d'un tel rapport, le fait qu'il existe est important pour la conception hérodotéenne de l'histoire.

66. I 169,2, cf. supra n. 5. Indépendamment de la réalité historique de cette soumission, il est important de remarquer qu'Hérodote sent le besoin de dire que les insulaires sont soumis à Cyrus, de souligner donc une rupture de l'équilibre déjà sous ce dernier. 
capturés. Ils n'ont pas voulu danser avant, ils danseront après avoir été conquis.

Les Ioniens et Éoliens, "poissons" insulaires, continuent de se faire "ratisser" à différents moments des Histoires; on aurait bien appliqué ce procédé aux Grecs de la côte d' $\Lambda$ sie, s'il n'y avait eu des difficultés techniques; en revanche, ce langage spécifique, lié à la pêche, n'est pas utilisé pour les autres peuples, habitants des îles ou des côtes de l'Égée, qui entrent en contact avec les Perses.

L'épisode de Sestos, enfin, marque un retour à l'équilibre, par la séparation des deux continents: les ponts sont rompus, les poissons revivent. La résurrection des poissons salés doit être lue sur deux plans: en effet, leur retour à la vie est explicitement mis en rapport par Artayctès avec la vengeance de Protésilas; et le héros lié à la guerre de Troie permet de rattacher la fin des Histoires à leur début, en particulier aux paragraphes sur les rapts des femmes, puisque justement la guerre de Troie, conduite à cause d'une seule femme, avait constitué la première grande injustice ${ }^{67}$. A la fin des Histoires, le dernier Perse faisant partie de l'expédition de Xerxès, Artayctès, est puni pour sa transgression contre le premier Grec tué dans la Guerre de Troie; l'anecdote à propos de Protésilas sert ainsi à fermer le cercle temporel qui unit les deux grandes invasions transcontinentales ${ }^{68}$.

Mais la résurrection des poissons à la fin des Histoires est aussi en rapport avec l'apologue de Cyrus: les "poissons Ioniens" dansent et se vengent au moment où la lutte va recommencer, après Mycale et après la libération des îles. Nous sommes d'autant plus autorisés à lire la résurrection des poissons sur deux plans -et non pas seulement en rapport avec Protésilasque l'interprétation mettant le prodige en relation avec Protésilas est donnée par Artayctès, qui lit les événements en rapport avec son expérience personnelle; Hérodote ne refuse ni n'appuie cette interprétation, plus encore, à aucun moment il n'est dit explicitement qu'un des Grecs la partage.

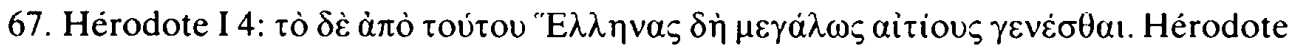
attribue cette opinion aux logioi perses. Cf. infra $n .73$.

68. Comme l'a récemment montré D. Boedeker, "Protesilaos and the End of Herodotus' Histories", Classical Antiquity, 7, 1988, pp. 30-48. Voir aussi Nagy, op. cit., pp. 268-273, sur Protésilas en tant que $\sigma \tilde{\eta} \mu \alpha$ : son "signe" s`adresse à Artayctès, mais aussi

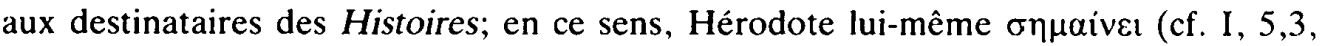
cit.infra, n. 73). En nous rattachant à cette interprétation, nous proposerons un triple référent pour le $\sigma \tilde{\eta} \mu \alpha$ donné par Protésilas: une relation immédiate avec Artayctès; une relation plus vaste avec la structure des Histoires; enfin un référent externe au texte, lié au contexte dans lequel vivent Hérodote et son public. 
Les poissons salés renvoient donc aussi bien à la momie de Protésilas qu'aux Grecs d'Asie Mineure. La situation de ces derniers au moment de l' $\alpha \bar{i}$ vo $\zeta$ et à la fin des Histoires est la même; avec Crésus, il y avait eu un accord avec les insulaires (et si l'on admet qu'Hérodote partage l'opinion des Perses selon laquelle l'Asie leur appartient, avec la soumission du continent et l'indépendance des îles la situation est stable). Au contraire, l'aĩvos de Cyrus est donné en un moment où les Grecs des îles sont encore libres et où ceux du continent le sont redevenus, puisqu'ils ne sont plus sous le contrôle des Lydiens et que Cyrus ne les a pas encore soumis. Mais la Perse se prépare à les soumettre, et la soumission est accomplie en I

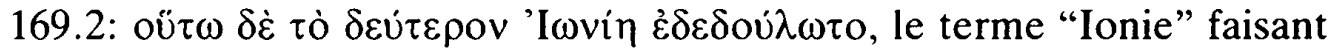
référence aux Grecs de la côte; les insulaires se soumettent tout de suite après, et il s'agit pour eux de la première réduction en esclavage ${ }^{69}$.

Des poissons réapparaissent implicitement avec la $\sigma \alpha \gamma \eta \dot{v \varepsilon v \sigma ı \varsigma ~ d e s ~ i ̂ l e s, ~}$ conséquence de la révolte d'Ionie; là aussi, l'écrasement par les Perses ${ }^{70}$ fait suite à un moment de liberté.

Enfin, avec Artayctès, nous sommes après la bataille de Mycale,

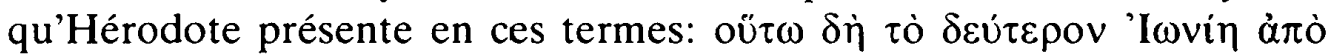
$\Pi \varepsilon \rho \sigma \dot{\varepsilon} \omega \nu \dot{\alpha} \pi \dot{\varepsilon} \sigma \tau \eta(\mathrm{IX} 105)^{71}$. A ce moment, les poissons revivent et les Io-

69. Dans cette optique, Cyrus apparaît comme le premier vrai perturbateur. Il est vrai qu'après la section sur les rapts Hérodote, affirmant qu'il indiquera celui qui à sa connaissance a le premier fait des torts à des Grecs, commence à raconter la conquête de Crésus. Ce dernier pourtant présente plusieurs traits qui empêchent de le voir dans le cadre du choc entre les deux continents, cf. le fait qu'il cherche à conclure une alliance avec les Grecs d'Europe, les dons qu'il fait à Delphes, et I 6,2: "Ce Crésus le premier des Barbares à notre connaissance, asservit certains Grecs qu'il obligea à lui payer tribut et se fit des amis de certains autres". Voir à ce propos P. Tozzi, La rivolta ionica, Pisa 1978, p. 46 et p. 115, qui souligne que seulement avec l'arrivée de Cyrus se pose de façon forte le problème de la liberté des Grecs d'Ionie, comme le montre le refus des Ioniens de se rebeller à Crésus. malgré les exhortations de Cyrus, Hérodote, I, 76, 3; cf. aussi l'analyse très nuancée de M. Lombardo, "Erodoto storico dei Lidî", in Hérodote et les peuples non-Grecs, Entretiens Hardt XXXV, Vandœuvres-Genève 1990, pp. 171-203, en part. pp. 119-201. Contra, Nagy, op.cit., pp. 229-230 et n. 76.

70. Cf. VI, 32: “Ainsi les Ioniens furent pour la troisième fois réduits en servitude ("I $\omega v \varepsilon \varsigma \kappa \alpha \tau \varepsilon \delta o u \lambda \omega \theta \eta \sigma \alpha v$ ); ils l'avaient été une première fois par les Lydiens, et cela faisait alors deux fois de suite qu'ils l'étaient par les Perses". Toutefois, la révolte avait

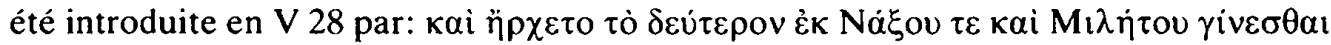
$\kappa \alpha \kappa \dot{\alpha}$, où "pour la deuxième fois" renvoie, en le caractérisant comme le premier malheur, à la soumission de l'Ionie par Cyrus; Crésus ici encore est laissé de côté.

71. Le renvoi à la révolte d'Ionie est évident. Hérodote, IX, 106, avec le refus des Athéniens d'évacuer l'Ionie, selon la proposition des Péloponnésiens, montre que la lutte vient juste de commencer, et qu'elle va se poursuivre. 
niens regagnent la liberté; à ce moment aussi, Hérodote arrête sa narration $^{72}$. De la sorte, la résurrection des poissons au moment de la capture d'Artayctès lui permet, par sa double référence à Protésilas et à la conquête de l'Ionie par Cyrus, de fermer en même temps le cercle des injustices "mythiques" et le cercle des injustices "historiques" 73 .

\section{Conclusion}

Il est possible de faire encore une dernière remarque concernant la façon de travailler d'Hérodote et l'historicité de l'al̃vo comparaison avec la version des événements qui nous a été transmise par Diodore nous permet d'aller encore un peu plus loin. Diodore nous donne une version différente de celle d'Hérodote: les Ioniens envoient toujours l'ambassade à Cyrus, mais c'est Harpagos qui leur répond, avec un aĩvo différent, visant toutefois à la même signification:

Harpagos avait été désigné par Cyrus stratège des territoires près de la mer, et lorsque les Grecs d'Asie envoyèrent une ambassade auprès de Cyrus pour conclure une alliance, il leur fit remarquer qu'ils faisaient quelque chose de très ressemblant à ce qui lui était arrivé à lui-même auparavant. En effet, voulant se marier, il demanda au père la jeune fille; mais celui-ci ne le jugeant pas au début digne du mariage fiança sa fille à un homme plus puissant; par la suite, voyant qu'Harpagos était honoré par le roi, il voulut lui

72. Le dernier mot des Histoires est $\delta o u \lambda \varepsilon v ́ \varepsilon ı v$ : les Perses choisissent de ne pas devenir "esclaves d'autrui en cultivant de grasses plaines", IX, 122. Or, le verbe $\delta$ ou $\lambda \varepsilon v i \varepsilon ı$, qui scande l'histoire des Grecs d'Asie Mineure depuis la conquête de Cyrus jusqu'à la répression de la révolte d'Ionie par Darius, n'est pas utilisé dans la narration pour décrire la conquête de l'Ionie par Crésus (si ce n'est de façon implicite en VI 32, cf. supra n. 69): le terme employé est $\kappa \alpha \tau \alpha \sigma \tau \rho \varepsilon \dot{\phi} \omega$, cf. Hérodote I 6,2 et I 27.1 ; I 29,1. Une seule fois,

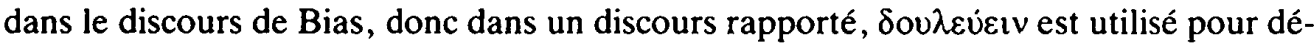
signer la situation des Grecs de la côte sous Crésus, Hérodote I 27,4. Le vocabulaire utilisé semble donc confirmer ce que nous avançons à la n. 69 supra.

73. Ce qui correspond à sa prise de position au début des Histoires: "Quant à moi, je ne vais pas prononcer, à propos de ces événements [les rapts de femmes], qu'il en fut ainsi ou d'une autre façon. J'indiquerai celui qui, autant que je sache personnellement, a pris le premier l'initiative d'actes offensants envers les Grecs", Hérodote, I, 5,3: $\tau$ òv $\delta \dot{\varepsilon}$

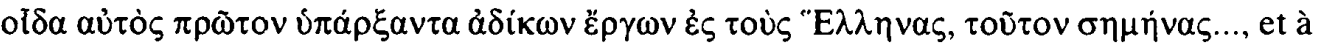
la séparation entre temps des dieux et temps des hommes, telle que l'a esquissée par exemple C. Darbo-Peschanski, Le discours du particulier. Essai sur l'enquête hérodotéenne, Paris, 1987, pp. 25-38. On peut ajouter que l'interprétation reliant le prodige à Protésilas est attribuée à un Perse, tout comme ce sont les opinions des logioi perses à propos des rapts de femmes qui sont rapportées au début $(I, 1,1 ; 1,2,1 ; I, 4,3 ; \mathrm{I}$, $5,1 ; \mathrm{I}, 5,3)$. 
donner sa fille. Mais lui répondit qu'il ne la voulait plus comme femme; il pourrait toutefois accepter de la prendre comme concubine. (Diod. IX 35).

La morale qui suit est la même que dans le texte d'Hérodote: les Grecs n'ont pas voulu être amis des Perses lorsque Cyrus le leur a demandé; maintenant que la situation a changé, Cyrus voudra tout au plus les recevoir comme esclaves. Il est évident que la version de Diodore est plus banale, "normalisante", moins chargée de signification.

Comment expliquer l'existence de ces deux versions parallèles? On peut imaginer qu'au moment où Hérodote composait les Histoires, plusieurs anecdotes devaient circuler en Ionie, liées aux Sept Sages et à leurs rapports avec les puissances lydienne et perse ${ }^{74}$ ou plus généralement aux démêlés entre Lydiens, Perses et Grecs. La fable "ésopique" du flûtiste et des poissons pouvait déjà, dans la tradition ionienne sur la conquête perse, avoir été utilisée pour illustrer la réponse de Cyrus aux Ioniens; de même la version rapportée par Diodore pouvait aussi déjà exister. On peut renvoyer à ce propos à ce qu'Hérodote lui-même nous dit avant de commencer à raconter l'histoire de Cyrus: "Je suivrai dans mon exposé ce que disent quelques-uns des Perses, ceux qui ne veulent pas magnifier l'histoire de Cyrus mais dire la vérité, tout en étant capable de faire connaître aussi sur le compte de Cyrus trois autres versions différentes"75. Hérodote n'aura donc eu qu'à opérer un choix. Entre les versions possibles, il a choisi celle qui était la plus chargée de signification. La connaissance de la tradition sur la $\sigma \alpha \gamma \dot{v} v \varepsilon \sigma \iota \varsigma$ des Ioniens et Éoliens permettait de lire toutes ces traditions comme un ensemble cohérent, tout en justifiant, d'un point de vue strictement historique ${ }^{76}$, le fait d'attribuer à un Perse un tel dis-

74. Plusieurs anecdotes nous sont transmises de façon presque identique par Hérodote et Diodore; nous en avons qui ne sont transmises que par un de ces auteurs; nous avons aussi des cas où l'anecdote est attribuée, par Hérodote ou Diodore ou par la tradition, à l'un ou l'autre des Sages (cf. Hérodote, I, 27, Bias ou Pittacos; même hésitation en Diodore IX 25; mais là Diodore tire peut-être son renseignement d'Hérodote). Tout cela montre qu'il y a eu un foisonnement de variantes. On peut imaginer comme contexte de formation de ces variantes le cadre décrit par Xénophane dans le fr. 13 G.P. = 22 D.-K.: des réunions hivernales, où, étendus auprès du feu, après avoir bien mangé, l'on se racontait des histoires, en posant des questions telles que "Quel âge avais-tu lorsque survint le Mède?". Sur le rôle du symposion dans la formation des traditions des Grecs d'Asie Mineure, voir M. Lombardo, art. cit., en part. pp. 185-186.

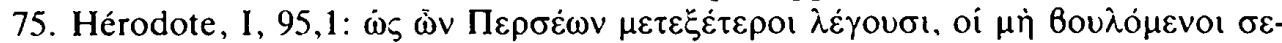

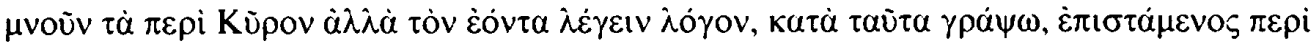

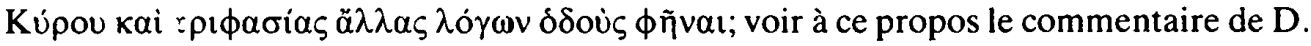
Asheri, Erodoto. Storie, I, Milano 1988.

76. Une telle préoccupation est d'ailleurs moderne, je ne crois pas qu'il faille l'at- 


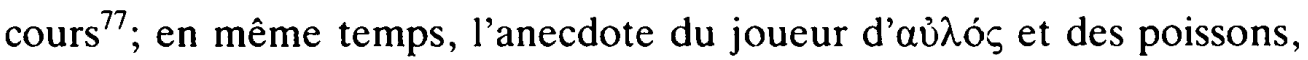
une fois mise en rapport avec la $\sigma \alpha \gamma \eta \dot{v \varepsilon v \sigma ı \varsigma, ~ r e ́ v e i l l a i t ~ t o u t e s ~ s o r t e s ~}$ d'échos dans la tradition grecque. En outre, Hérodote a fait en sorte d'insérer réponse de Cyrus et $\sigma \alpha \gamma \eta \dot{v \varepsilon v \sigma ı \varsigma ~ d a n s ~ l a ~ s t r u c t u r e ~ g l o b a l e ~ d e s ~} \mathrm{His}$ toires, en tissant un réseau de rapports à longue distance qui tient jusqu'à la fin de l'œuvre ${ }^{78}$. En ce sens, on peut parler de manipulation; mais une telle manipulation fonde nécessairement tout récit historique, puisque l'écriture de l'histoire ne peut se dispenser d'une organisation narrative, d'une mise en intrigue ${ }^{79}$. Et c'est justement cette mise en intrigue qui donne finalement son sens et sa raison d'être à l'apologue du flûtiste et des poissons.

L'aĩvos de Cyrus doit donc être lu sur plusieurs niveaux: il a tout d'abord la fonction narrative de relater la réponse de Cyrus, tout en donnant un enseignement plus général. La forme de l'aĩvos qu'Hérodote choisit pour relater la réponse de Cyrus donne à celle-ci une fonction performative; elle pousse en même temps le lecteur-auditeur à chercher une signification sur un autre niveau. Cet $\alpha$ İvo $\varsigma$ peut être compris sur la base de la tradition grecque: nous avons vu que l'Odyssée compare les prétendants à des poissons pris au filet et en train de mourir lentement; et que Sarpédon dans l'Iliade parle d'un filet qui pourrait envelopper et détruire les

tribuer à Hérodote; la cohérence suffit à prouver la "vérité". Je me limite à renvoyer à $O$. Murray, art. cit., en part. pp. 100-101, et p. 107: "We must recognize that ultimately truth in Herodotus is a question of aesthetics and morality, as much as of fact".

77. Je laisse de côté le problème de savoir si Hérodote pouvait en outre, par ses informateurs, avoir connaissance de l'idéologie qui transparaît par exemple des textes assy-

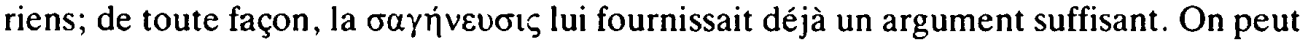
tout de même remarquer que l'information d'Hérodote sur la Perse semble bonne; $\mathrm{P}$. Tozzi, "Erodoto V, 106: nota preliminare sull'insurrezione ionica", Athenaeum, 53, 1975, pp. 136-147, montre par exemple que le discours d'Histiée à Darius correspond de près à l'idéologie qui s'exprime dans les inscriptions de Darius et de Xerxès. Voir aussi, pour une évaluation du rapport entre les renseignements fournis par Hérodote et les sources perses, notamment les tablettes de Persépolis, D.M. Lewis, "Persians in Herodotus", in The Greek Historians. Literature and History, Papers presented to A.E. Raubitschek, Stanford, 1985, pp. 101-117.

78. Réseau produisant, par l'interaction de ses composants, une signification qui correspond à l'horizon d'attente idéologique des contemporains d'Hérodote: l'opposition entre pouvoir maritime et pouvoir continental, la situation des insulaires, les problèmes de la frontière ionienne avec la Perse sont à la base des préoccupations des Grecs du V siècle, des Athéniens en particulier, exhortés à devenir des "insulaires" afin de pouvoir garder leur liberté et leur empire (Thucydide, 1, 143,5).

79. Je renvoie une fois de plus à Payen, art.cit., pp. 527-529 et pp. 542-544. 
Troyens, image reprise par Eschyle dans l'Agamemnon pour décrire la prise de Troie, et dans les Perses pour raconter la défaite des barbares. Or nous sommes, dans le cas de la guerre de Troie comme dans celui des Guerres médiques, toujours à l'intérieur d'un schéma opposant les deux continents.

D'autre part, la tradition orientale, par le témoignage des inscriptions assyriennes, des textes de l'Ancien Testament et du texte même d'Hérodote, nous donne pour l'aĩvos de Cyrus un référent: pour Hérodote et pour ses contemporains, comme pour nous d'ailleurs, la réponse de Cyrus répond à des critères de vraisemblance. Le discours de surface des Histoires, discours qui relate des événements, apparaît répondre, dans ce cas précis, de façon étonnamment correcte à des critères d'historicité; et pourtant, initialement, précisément cette réponse de Cyrus aurait pu sembler, par ses rapports avec la fable ésopique, totalement construite.

Enfin, l'aivos que Cyrus adresse aux Ioniens joue un rôle dans l'organisation générale des Histoires, puisqu'on peut le mettre en rapport avec la

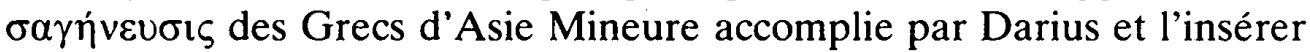
dans le gigantesque $R$ Ring reliant le début et la conclusion de l'œuvre ${ }^{80}$. Au moyen de cette misc cn pcrspective I Iérodote adresse à son tour son aĩvos, son message, aux lecteurs-auditeurs des Histoires.

(Université de l'Aquila)

Paola CECCARELLI

80. Voir par exemple H. Bischoff, "Sinn des letzten Kapitels", (tiré de H. Bischoff, Der Warner bei Herodot, Borna-Leipzig 1932, pp. 78-83) in W. Marg, Herodot, Darmstadt 1962, pp. 670-676; J. Cobet, Herodots Exkurse und die Frage der Einheit seines Werkes, Wiesbaden 1971, pp. 171-176, avec d'autres renvois; et J. Herington, "The Closure of Herodotus'Histories", Illinois Classical Studies, 16, 1991, pp. 149-160. 